Original Review Paper

\title{
Multi-Timescale Microscopic Theory for Radiation Degradation of Electronic and Optoelectronic Devices
}

\author{
${ }^{1}$ Danhong Huang, ${ }^{2}$ Fei Gao, ${ }^{1}$ D.A. Cardimona, ${ }^{1}$ C.P. Morath and ${ }^{1}$ V.M. Cowan \\ ${ }^{1}$ Air Force Research Laboratory, Space Vehicles Directorate, Kirtland Air Force Base, NM 87117, USA \\ ${ }^{2}$ Department of Nuclear Engineering and Radiological Sciences, \\ University of Michigan, 500 S. State Street, Ann Arbor, Michigan 48109, USA
}

Article history

Received: 13-01-2015

Revised: 21-02-2015

Accepted: 29-04-2015

Corresponding Author:

Danhong Huang

Air Force Research Laboratory,

Space Vehicles Directorate,

Kirtland Air Force Base, NM

87117, USA

Email: danhonghuang1647@msn.com

\begin{abstract}
A multi-timescale hybrid model is proposed to study microscopically the degraded performance of electronic devices, covering three individual stages of radiation effects studies, including ultra-fast displacement cascade, intermediate defect stabilization and cluster formation, as well as slow defect reaction and migration. Realistic interatomic potentials are employed in molecular-dynamics calculations for the first two stages up to $100 \mathrm{~ns}$ as well as for the system composed of layers with thicknesses of hundreds of times the lattice constant. These quasi-steady-state results for individual layers are input into a ratediffusion theory as initial conditions to calculate the steady-state distribution of point defects in a mesoscopic-scale layered-structure system, including planar biased dislocation loops and spherical neutral voids, on a much longer time scale. Assisted by the density-functional theory for specifying electronic properties of point defects, the resulting spatial distributions of these defects and clusters are taken into account in studying the degradation of electronic and optoelectronic devices, e.g., carrier momentum-relaxation time, defect-mediated non-radiative recombination, defect-assisted tunneling of electrons and defect or charged-defect Raman scattering as well. Such theoretical studies are expected to be crucial in fully understanding the physical mechanism for identifying defect species, performance degradations in field-effect transistors, photo-detectors, light-emitting diodes and solar cells and in the development of effective mitigation methods during their microscopic structure design stages.
\end{abstract}

Keywords: Radiation Effects in Semiconductor Devices, RadiationInduced Defects in Semiconductors, Radiation-Induced Degradation of Semiconductor Device Performance

\section{Introduction}

Point defects (vacancies and interstitial atoms) are produced by the displacements of atoms from their lattice sites, (Was, 2007; Sigmund, 2006) where the atom displacements are mainly induced by a Primary Knockout Atom (PKA) on a time scale shorter than 50 ps. This initial phase is followed subsequently by a defect reaction (clustering or dissolution of clusters), (Devanathan et al., 2001) and further by the thermally activated migration (Posselt et al., 2005) of the point defects and defect clusters over a time scale longer than $100 \mathrm{~ns}$. The combination of all these processes, resulting in a significant concentration of surviving defects in the crystal, is physically termed particle irradiation displacement damage (in addition to the well know $\gamma$-ray electron ionization cascade damage). Such radiation displacement damage effects depend not only on the energy-dependent flux of the incident particles (protons, neutrons, ions, etc.) but also on the differential energy transfer cross sections (probabilities) for collision between atoms, interatomic Coulomb interactions and kinetic-energy loss to electrons inside an atom. Irradiation temperature also significantly affects the motion of defects, their stability as clusters and the formation of Frenkel pairs (Gao and Weber, 2003a).

On the other hand, electron devices are usually classified either as electronic ones, where electrons 
respond to an applied voltage as a current flow, or optoelectronic ones, in which electrons perform interband/intraband optical transitions in the presence of an incident light signal. (Gumbs and Huang, 2013) For an electronic device (e.g., field-effect transistors in an integrated circuit), the momentum-relaxation time of electrons, due to scattering by randomly-distributed defects, plays a crucial role in determining the electron mobility, (Strour et al., 2003) while the photo-excited electron lifetime, due to nonradiative recombination with defects, is proven to be a key factor affecting the sensitivity or the performance of optoelectronic devices (e.g., photo-detectors and lightemitting diodes) (Weatherford and Anderson, 2003).

In a perfect crystal, the continuous free-electron states are quantized into many Bloch bands separated by energy gaps and these Bloch electrons move freely inside the crystal with an effective mass different from that of the free electrons (Callaway, 1991). In the presence of defects, however, the field-driven current flow of Bloch electrons in a perfect crystal will be scattered locally by these defects, leading to a reduced electron mobility. In addition, photo-excited Bloch electrons could acquire a shortened lifetime, giving rise to a degraded quantum efficiency due to enhanced non-radiative recombination with defects. The dangling bonds attached to the point defects may capture extra electrons to form charged defects. In this case, the positively-charged holes in the system will be trapped to produce a strong spacecharge field, while the negatively-charged electrons may generate the so-called $1 / \mathrm{f}$-current noise in their chaotic motion due to the presence of many potential minima and maxima from randomly-distributed charged defects.

Point defects in crystals, as shown in Fig. 1, can be generated by particle irradiation in both bulk and nanocrystals composed of many grains with different sizes (Gao et al., 2010). One of the effective calculation methods for studying the non-thermal spatial-temporal distributions of radiation-induced point defects is the Molecular-Dynamics (MD) model based on a stepped time-evolution approach (also termed the collisional and thermal spike stages), which involves the total force by summing over the interatomic potentials from all the atoms in a finite system (Gao et al., 2009). The lattice vibration at finite temperatures can be taken into account by an initial thermal-equilibrium state for atoms (intrinsic vacancies and interstitials) in the system plus an initial velocity for one of the atoms in a specific direction. The system size increases quadratically with the initial kinetic energy of particles and the time scale runs up to several hundred picoseconds (called the quenching stage). Therefore, the defect reaction process by thermal migration cannot be included in this MD model due to its much longer time scale, although the other processes, such as displacement of lattice atoms, energy dissipation, spontaneous recombination and clustering, can be fully taken into account. If the system time evolution goes beyond $100 \mathrm{ps,}$ the kinetic lattice Monte-Carlo method can also be used (Rong et al., 2007). However, if the time scale exceeds several hundreds of nanoseconds (also called the annealing stage), the rate theory (Maksimov and Ryazanov, 1980; Golubov et al., 2012) has to be called in for studying the steady-state properties of the surviving defects (up to hours or days or even months).

We know that the MD model with a realistic interatomic potential has been developed for studying the nonthermal spatial-temporal distributions of radiation-induced point defects in noble transition metals and alloys and the density-functional theory has been widely used for calculating electronic properties of defects with pre-assumed specific defect configurations. On the other hand, a quantummechanical model has been well established for investigating defect effects on semiconductor electronic devices in the presence of spatially-uniform and randomly distributed point defects. However, to the best of our knowledge, no first-principle model and theory has been proposed so far to study microscopically the degraded performance of electronic devices induced by particle irradiation displacement damage.

Therefore, the theory presented in this study is expected to be very important in understanding the full mechanism for characterizing defects, performance degradations in transistors, photo-detectors, lightemitting diodes and solar cells, as well as in developing effective mitigation in early design stages. Equipped with our current multi-timescale microscopic theory, the experimental characterization of post-irradiated test devices will result in useful information on the device architecture's susceptibility to space radiation effects (we already know details on particle irradiation sources). On the other hand, the model should allow prediction of devices degradation on the basis of space weather forecast for the particular orbit.

Some of the equations presented below will be well-known to researchers in the materials science field, however, researchers in the device physics field may not be aware of them. With this paper, we hope to bridge the gap between researchers studying radiation-induced damage in materials and researchers studying radiation-induced performance degradation in devices. This should allow the formalism developed for the investigation of radiation-induced structural defects in nuclear reactor materials to be extended to the investigation of device performance degradation effects induced by particle radiation found in spacebased systems. 


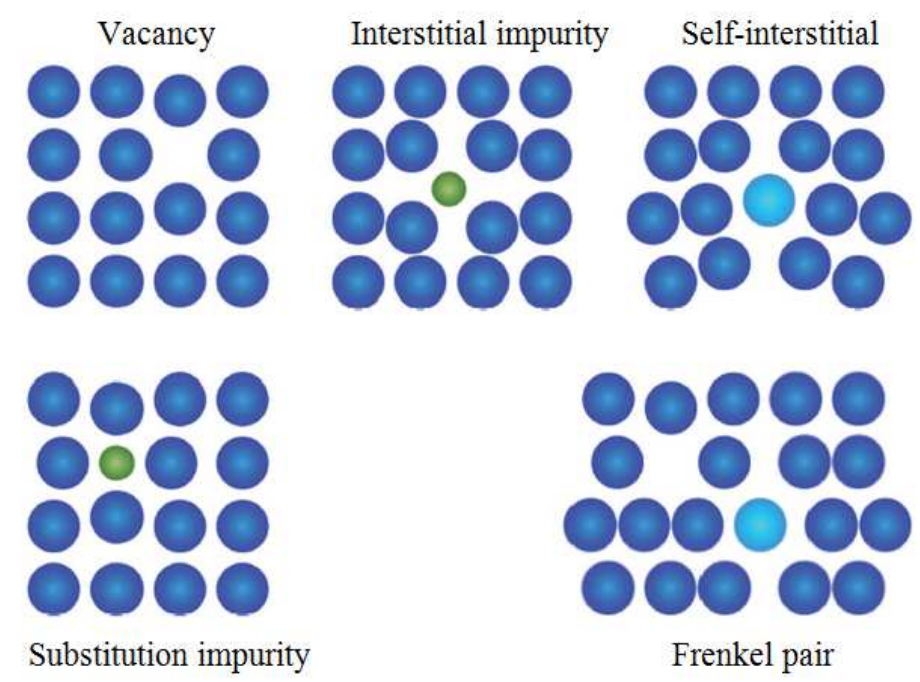

Fig. 1. Two-dimensional illustrations of different types of point defects in a crystal

The rest of the paper is organized as follows. In section II, we present our atomic-scale MD model to cover both the ultra-fast defect generation and intermediate defect stabilization stages, as well as the mesoscopic-scale rate theory for defect migration and interaction processes. In Section III, master equations for both planar dislocation-loop and spherical void growth are introduced for studying surface and bulk sink dynamics, respectively. In section IV, master equations are presented for exploring the steady-state spatial distribution of defects in layered structure materials. In addition, a density-functional theory is introduced for specifying electronic properties of point defects and four device physics models are employed for characterizing and understanding defect-assisted resonant tunneling, reduced carrier mobility, non-radiative recombination with defects and inelastic light scattering by charged defects. In section $\mathrm{V}$, an indication of how this defect theory might be applied to experimental data describing device performance degradation is presented. Finally, some concluding remarks are presented in section VI.

\section{Model and Theory}

\section{Atomic-Scale Modeling for Ultrafast Defect Generation (Displacement Cascade: $t<100$ ps)}

A schematic of a displacement-cascade event by proton irradiation is shown in Fig. 2. For the neutronnucleus elastic collision, this process can be simply regarded as colliding hard spheres as an approximation due to their charge neutrality. The more complicated inelastic collision of neutrons with a nucleus, however, could involve generating an additional neutron [(n, 2n)process] or photon emission [(n, $\gamma)$-process], which are both important to the displacement of atoms. For the proton-nucleus elastic collision, on the other hand, the extra interaction (potential function) between the electron cloud and the proton should be considered.

Commonly, the end product of a particle collision results in the PKA having an excess kinetic energy and the subsequent atom-atom interaction represents the most fundamental physical mechanism of the radiation displacement damage (Gao and Weber, 2000a; Gao and Weber, 2003b). Since radiation damage events are random in nature, a large number of damage events are required to obtain good statistics by choosing different directions and locations for the PKA. On the other hand, the dynamics in the damage procedure can be accurately described by employing a realistic interatomic potential (MD model). For incident charged particles, the detailed form of the interatomic potential depends on the closest separation between two collision partners, which itself is determined by the kinetic energy of the incident particles (e.g., heavy-slow ions and relativistic electrons).

The point defect generation as a result of displacement cascades is closely related to the PKA energy, which can be described statistically by an average transfer energy to the PKA. Such an average transfer energy can be calculated by using the energyloss theory and measured by the so-called proton (electron) energy-loss spectroscopy (Gumbs, 1989) as a function of various incident charged particle energies. The defects can also be identified experimentally by using positron annihilation (Tuomisto and Makkonen, 2013). With help from the computed energy loss of incident particles per unit length (called the loss function), the range of the particle before its full stop inside a crystal can be found. On the other hand, the MD method has been widely employed to simulate defect generation in a number of semiconductors, including $\mathrm{Si}$, (Rubia and Gilmer, 1995) SiC, (Gao and Weber, 2000) GaAs, (Nordlund et al., 2001) and GaN (Nord et al., 2003). 


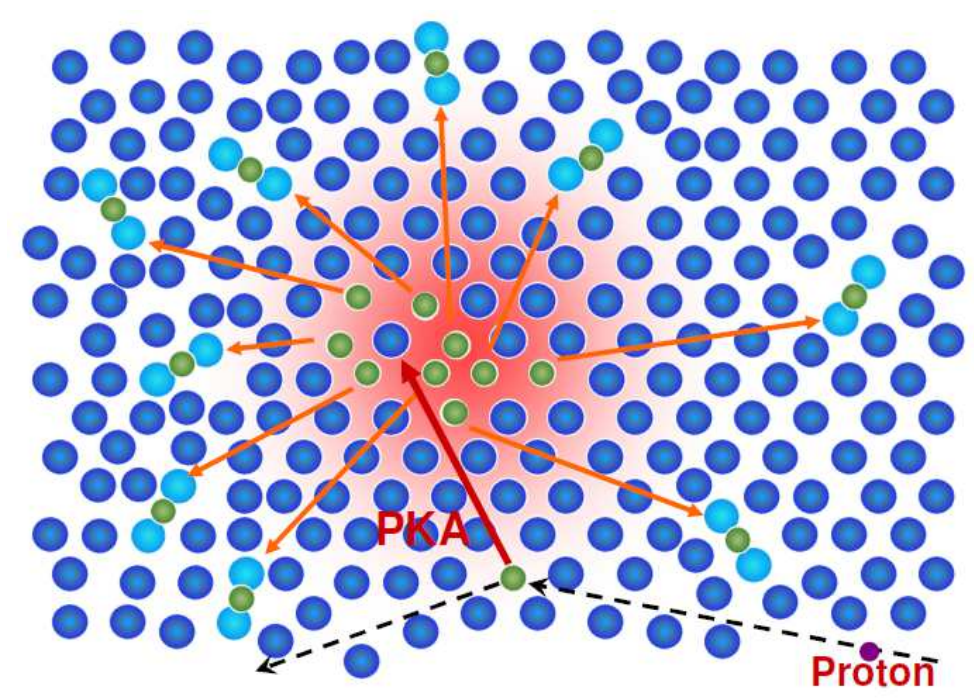

Fig. 2. Two-dimensional schematic of a displacement cascade induced by incident protons on a crystal

These simulations provide important insights into the mechanisms for defect generation in semiconductors and predict the number and type of defects, spatial distribution of defects and initial correlation among defect species produced by the incident radiation for subsequent device level models.

Basically, in MD simulations the time evolution of a set of interacting particles is tracked via the solution of Newton's equations of motion as shown below:

$$
F_{j}(t)=m_{j} \frac{d^{2} r_{j}(t)}{d t^{2}}
$$

where, the indices $j=1,2, \ldots, N$ label individual $N$ particles in the system, $r_{j}(t)\left[x_{j}(t), y_{j}(t), z_{j}(t)\right]$ is the position vector of the jth particle and $F_{j}(t)=-\sum_{k \neq j} \nabla_{j} V_{j k}$ is the force acting upon the jth particle at time $t$ with interacting potential $V_{j k}$ between the jth and kth particle and $\mathrm{m}_{\mathrm{j}}$ is the mass of the corresponding particle. In general, $F_{j}(t)$ will depend on both particle positions and velocities at time $t$. Here, the energy loss of protons to electrons is ignored due to thin samples considered. To integrate the above second-order differential equations, the instantaneous forces acting on the particles and their initial positions and velocities need to be specified. Due to the many-body nature of the problem, the equations of motion have to be discretized and solved numerically. The MD trajectories are defined by both position vector $\mathrm{r}_{\mathrm{j}}(\mathrm{t})$ and velocity vector $v_{j}(t)=\frac{d r_{j}(t)}{d t}$ and they describe the time evolution of the system in position-velocity phase space. Accordingly, the positions and velocities are propagated with a small time interval $\Delta t$ using numerical integrators. The numerical integration of
Newton's equations of motion is employed to find an expression that defines positions $r_{j}(t+\Delta t)$ at time $t+\Delta t$ in terms of the already known positions $r_{j}(t)$ at time $t$. Because of its simplicity and stability, the Verlet algorithm is commonly used in MD simulations. (Frenkel and Smit, 2002) However, other popular algorithms, such as Leapfrog, Velocity Verlet, Beeman's algorithms, (Frenkel and Smit, 2002; Allen and Tildesley, 1987) predictor-corrector, (Gear, 1971) and symplectic integrators, (Tuckerman and Martyna, 2000) are also widely adopted. For non-PKA particles, their two initial conditions can be set as $r_{j}(-\Delta t)=r_{j}(0)=R_{j}$, where $R_{j}$ is the lattice vector for the jth site. If the PKA is given an initial velocity $v_{0}$, in addition to $r^{P K A}(0)=r_{0}^{P K A}$ this leads to another initial condition $r^{P K A}(-\Delta t)=r_{0}^{P K A}-v_{0} \Delta t$.

In MD simulations, the atomic force field is crucial to determine physical systems in which collections of atoms are kept together by interatomic forces that can be calculated from empirical or semi-empirical interatomic potentials. Because of extensive applications of MD methods in materials science, a variety of techniques have been utilized over the years to develop reliable atomic-potential models. One of the early successful attempts to include manybody effects was the introduction of the embedding functional, (Norskov and Lang, 1980) which depends nonlinearly upon the coordination number (defined below) of each atom. This development led to the birth of the Embedded Atom Method (EAM), (Finnis and Sinclair, 1984) which provides a relatively accurate description for noble transition metals as well as their alloys. However, the Tersoff potential formalism (Tersoff, 1988) is based on the concept of bond order and has been widely applied to a large number of semiconductors. Novel many-body forms have been tried in an attempt to capture as much 
as possible the physics and chemistry of the bonding. A typical analytical form is constituted by a number of functions, depending on geometrical quantities, such as distances or orientations, or on intermediate variables, such as atom coordinations. For example, a Tersoff potential has the appearance of a pair potential as below:

$V=\frac{1}{2} \sum_{i, j=1}^{N} V_{i j}=\frac{1}{2} \sum_{i, j=1}^{N} \phi_{R}\left(r_{i j}\right)+\frac{1}{2} \sum_{i, j=1}^{N} B_{i j} \phi_{A}\left(r_{i j}\right)$

where, the terms with $i=j$ are excluded in the above summations, $r_{i j}=\left|r_{i}-r_{j}\right|$ and the first and second terms represent repulsive and attractive interactions, respectively. However, the second term in Equation (2) is not a true pair potential since $B_{i j}$ is not a constant. In fact, it is the bond order for the bond joining the ith and jth atoms and it is a decreasing function of a "coordination" $G_{i j}$ assigned to the bond. Therefore, we have $B_{i j}=B\left(G_{i \mathrm{j}}\right)$ and $G_{i j}$ is in turn defined by:

$$
G_{i j}=\sum_{k} f_{c}\left(r_{i k}\right) g\left(\theta_{i j k}\right) f_{c}\left(r_{j k}\right)
$$

where, $f_{c}(r)$ and $g(\theta)$ are suitable functions. The basic idea is that the $i-j$ bond is weakened by the presence of other $i-k$ and $j-k$ bonds involving the intermediate atom at site $k$. The amount of bond weakening is determined by where the other bonds are. Angular terms appear necessary to construct a realistic model. When using a potential, the researcher should always be familiar with its chemical transferability properties, such as bond length, bond angle and bond energy and validate critically the results obtained in unusual conditions, for example, for very low coordinations, very high temperature, or very high pressure.

\section{Atomic-Scale Modeling for Intermediate Defect Stabilization (Stable Defect and Cluster Formations: $100 \mathrm{ps}<\boldsymbol{t}<10 \mathrm{~ns}$ )}

The atom-displacement-generated point defects (vacancies and interstitial atoms) under particle irradiation will thermally diffuse in space and interact and react with dynamically distributed bulk sinks, planar dislocation loops, (Hirth and Lothe, 1982) spherical voids and clusters (due to collision cascade) at the same time. Generally, the kinetic energy of the incident particles (or equivalently, the recoil energy of the struck atom) determines the species and number of individual point defects during the initial phase (in addition to the rate of defect generation), while the flux of the incident particles decides the defect density and the nature of point-defect diffusion, (Mehrer, 2007) i.e., either in an independent way (for lowdensity non-interacting point defects) or in a direction-correlated way (for highdensity interacting point defects) (Posselt et al., 2008). The macroscopic property changes of the irradiated system are related to the particle energy-flux per unit time by the so-called damage function, or factor, which is extracted by experimental measurements. However, the damage factor is found to depend on the initial approximation in a sensitive way. Therefore, we are not able to treat physically the radiation displacement damage effects as a black box through a fitting procedure. Instead, we should understand the full dynamics of these defects on all time scales after they have been produced. The spatial distribution of the mobile Frenkel pairs (i.e., vacancy-interstitial pairs) that are created is crucial in determining the number that survive annihilation or immobilization by clustering due to damage cascade.

The statistically-averaged spatial distribution of point defects that are generated can be calculated based on the defect formation and recombination rates, as well as the follow-up processes for defect diffusion, interactions and reactions (Posselt et al., 2005). If the degree of atom displacements is limited due to high incident particle kinetic energies and low number intensities, we generally seek the radiation degradation effects on electronic and optoelectronic devices rather than looking at material level radiation damage effects when there is a significant level of atom displacements under intense low-energy particle irradiation (Strour et al., 2003). This radiation degradation depends not only on the particle radiation source and material, but also on the device structure and functionality. The analytical theory below can only provide a qualitative understanding of the collision- and thermally-activated diffusion processes, while the MD calculation based on a realistic interatomic potential is able to provide a quantitative result for comparison with experimental data.

\section{Point-Defect Generation Rate}

The spatially-temporally-dependent damage rate per unit volume for the displacement atoms in a crystal can be calculated from (Was, 2007):

$$
G_{0}(r, t)=n_{a t} \int_{E_{\min }}^{E_{\max }} d \varepsilon_{i} \sigma_{D}\left(r \mid \varepsilon_{i}\right) I_{e x t}\left(t \mid \varepsilon_{i}\right)
$$

where, $n_{a t}$ is the crystal atom volume density, $I_{e x t}\left(t \mid \varepsilon_{i}\right)$ represents the external dynamical energy-dependent particle intensity per unit energy, $\sigma_{D}\left(r \mid \varepsilon_{i}\right)$ stands for both the position- and energy-dependent displacement cross section and $E_{\min }\left(E_{\max }\right)$ corresponds to the minimum (maximum) kinetic energy in the energy distribution of incident particles.

Since the displacement cross section $\sigma_{D}\left(r \mid \varepsilon_{i}\right)$ in Equation (4) physically describes the probability for the displacement of struck lattice atoms by incident particles, we can directly write down: 


$$
\sigma_{D}\left(r \mid \varepsilon_{i}\right)=\int_{\varepsilon_{1}}^{\varepsilon_{2}} d \varepsilon_{R} \sigma_{C}\left(r \mid \varepsilon_{i}, \varepsilon_{R}\right) N_{D}\left(t \mid \varepsilon_{R}\right)
$$

where, $\sigma_{C}\left(r \mid \varepsilon_{i}, \varepsilon_{R}\right)$ is the differential energy transfer cross section by collision, which measures the probability that an incident particle with kinetic energy $\varepsilon_{i}$ will transfer a recoil energy $\varepsilon_{\mathrm{R}}$ to a struck lattice atom, $N_{D}\left(r \mid \varepsilon_{R}\right)$ represents the average number of displaced atoms due to collision and $\varepsilon_{l}\left(\varepsilon_{2}\right)$ labels the minimum (maximum) recoil energy acquired by the struck lattice atom. Although $N_{D}\left(r \mid \varepsilon_{R}\right)$ can be directly determined by MD simulation, (Terentyev et al., 2006) the simplest approximation to estimate the displaced atoms is the Kinchin-Pease model (for a solid composed of randomly arranged atoms by ignoring focusing and channeling effects). (Kinchin and Pease, 1955; Olander, 1976) In practice, $N_{D}\left(r \mid \varepsilon_{R}\right)$ can be accurately determined by either classic (Devanathan et al., 1998) or ab initio MD simulations (Gao et al., 2011).

In addition, the magnitude of the differential energy transfer cross section $\sigma_{C}\left(r \mid \varepsilon_{i}, \varepsilon_{R}\right)$ introduced in Equation (5), which can be regarded as the crystal response to an external particle collision with lattice atoms, depends on the detailed collision mechanism and the form of the scattering potential as well. Here, as a simple example, we first give the expression of the differential energy transfer cross section for the elastic scattering. For the well-known Rutherford elastic scattering model (Rutherford, 1911) based on an unscreened Coulomb potential $U_{R}(\rho)=$ $\mathrm{Z}_{1} \mathrm{Z}_{2} \mathrm{e} / \in_{0} \rho$ for protons with $\rho$ being the radius in the local frame centered on the lattice atom, we get:

$\sigma_{C}\left(r \mid \varepsilon_{i}, \varepsilon_{R}\right) \equiv \sigma_{R}\left(r \mid \varepsilon_{i}, \varepsilon_{R}\right)=\frac{\pi b_{0}^{2}(r)}{4} \frac{\varepsilon_{i} \gamma(r)}{\varepsilon_{R}^{2}}$

where, $\gamma(\mathrm{r})=4 \mathrm{mM}(\mathrm{r}) /[\mathrm{M}(\mathrm{r})+\mathrm{m}]^{2}, \mathrm{~m}[\mathrm{M}(\mathrm{r})]$ is the mass of the incident particle (surface atoms or different lattice atoms $), b_{0}(r)=Z_{1} Z_{2}(r) e^{2} / \eta(r) \in_{0} \varepsilon_{i}$ with $Z_{1}$ and $Z_{2}(r)$ being the nuclear charge numbers for particles and different lattice atoms and $\eta(r)=m /[M(r)+m]$.

If the kinetic energy of the incident particles is very high, the Rutherford scattering model becomes no longer applicable. In this case, we have to consider hard-sphere type collisions for neutrons, which leads to:

$\sigma_{C}\left(r \mid \varepsilon_{i}, \varepsilon_{R}\right) \equiv \sigma_{H S}\left(r \mid \varepsilon_{i}, \varepsilon_{R}\right)=\frac{\pi B^{2}(r)}{\gamma(r) \varepsilon_{i}} \operatorname{In}\left[\frac{A}{\eta(r) \varepsilon_{i}}\right]$

where, a Born-Mayer potential $\mathrm{U}_{\mathrm{B}-\mathrm{M}}(\rho)=\mathrm{A} \exp (-\rho / \mathrm{B})$ is employed (Abrahamson, 1969).

On the other hand, nuclear scattering with heavyslow ions represented by a power-law interacting potential $U_{I}(\rho)=\left(e / \in_{0} a_{B}\right)\left(Z_{1} / Z_{2}\right)^{5 / 6}\left(a_{B} / \rho\right)^{2}$ leads to:

$$
\begin{aligned}
& \sigma_{C}\left(r \mid \varepsilon_{i}, \varepsilon_{R}\right) \equiv \sigma_{I}\left(r \mid \varepsilon_{i}, \varepsilon_{R}\right) \\
& =\frac{4 E_{a}(r) \Lambda^{2}(r) \xi(r)}{\gamma(r) \varepsilon_{i}^{2}\left[1-4 \xi^{2}(r)\right]^{2} \sqrt{X(r)[1-X(r)]}}
\end{aligned}
$$

where, $X(r) \quad=\quad \varepsilon_{R} / \chi(r) \varepsilon_{i} \zeta(r)$
$=\cos ^{-1}[\sqrt{X(r)}] / \pi, \Lambda(r)=0.8853 a_{B} /\left[Z_{1} Z_{2}(r)\right]^{1 / 6}$ is the screening length with $\mathrm{a}_{\mathrm{B}}$ being the Bohr radius and $\mathrm{E}_{\mathrm{a}}(\mathrm{r})$ $=\left(\mathrm{e}^{2} / \epsilon_{0} \mathrm{a}_{\mathrm{B}}\right)\left[\mathrm{Z}_{1} / \mathrm{Z}_{2}(\mathrm{r})\right]^{7 / 6} / \eta(\mathrm{r})$.

In the special case, for the incidence of relativistic light electrons, we have:

$$
\begin{aligned}
& \sigma_{C}\left(r \mid \varepsilon_{i}, \varepsilon_{R}\right) \equiv \sigma_{I}\left(r \mid \varepsilon_{i}, \varepsilon_{R}\right)=\frac{\pi Z_{2}^{2} e^{4}}{\epsilon_{0}^{2} m_{0}^{2} c^{4}} \frac{1-\beta_{0}^{2}}{\beta_{0}^{4}} \\
& \times\left\{1-\beta_{0}^{2} \frac{\varepsilon_{R}}{E_{2}(r)}+\pi \frac{a_{0}(r)}{\beta_{0}}\left[\sqrt{\frac{\varepsilon_{R}}{E_{2}(r)}}-\frac{\varepsilon_{R}}{E_{2}(r)}\right]\right\} \frac{E_{2}(r)}{\varepsilon_{R}^{2}}
\end{aligned}
$$

where, $\beta_{0}=v / c$ with $v$ being the velocity of incident electrons, $E_{2}(r)=\left[2 \varepsilon_{i} / M(r) c^{2}\right]\left(\varepsilon_{i}+2 m_{0} c^{2}\right), m_{0}$ is the freeelectron mass and $\alpha_{0}(r)=Z_{2}(r) / 137$. Moreover, we have the relation $\beta_{0}^{2}=1-\left(m_{0} c^{2} / \varepsilon_{i}\right)^{2} \leq 1$ for the relativisticparticle velocity and kinetic energy.

For the case of isotropic inelastic scattering with an energy loss $Q_{0}$, we have the differential energy transfer cross section:

$$
\begin{aligned}
& \sigma_{C}\left(r \mid \varepsilon_{i}, \varepsilon_{R}\right) \equiv \sigma_{i n}^{\prime}\left(r \mid \varepsilon_{i}, \varepsilon_{R}\right) \\
& =\frac{\sigma_{i s}\left(r \mid \varepsilon_{i}, Q_{0}\right)}{\gamma(r) \varepsilon_{i}}\left[1+\frac{Q_{0}(r)}{\varepsilon_{i}} \frac{A(r)+1}{A(r)}\right]^{-1 / 2}
\end{aligned}
$$

where, $A(r)=M(r) / m$ and $\sigma_{i s}\left(r \mid \varepsilon_{i}, Q_{0}\right)$ represents the isotropic differential energy transfer cross section for the resolved resonance in the center-of-mass frame.

In addition, the incident-particle kinetic energy is no longer a constant if the particles are charged, e.g., protons and ions. In this case, we have $\varepsilon_{i} \rightarrow \varepsilon_{i}(z)=\left[\sqrt{\varepsilon_{0}}-k z / 2\right]^{2}$ with $\varepsilon_{0}$ being the incident-particle energy at the left boundary $\mathrm{z}=0$, where a layered structure in the $\mathrm{z}$ direction is assumed. As a result, we find that electronic stopping will dominate at short distances, while elastic collisions will dominate near the end of the range.

As an example, by using $\sigma_{C}\left(\mathrm{r} \mid \varepsilon_{i}, \varepsilon_{R}\right)$ from Equation (6) and $\mathrm{N}_{\mathrm{D}}\left(\mathrm{r} \mid \varepsilon_{\mathrm{R}}\right)$ from the Kinchin-Pease model, the displacement cross section from Equation (5) becomes:

$\sigma_{D}\left(r \mid \varepsilon_{i}\right) \approx\left[\frac{\gamma(r)}{4 E_{t h}(r)}\right] \sigma_{s}\left(r \mid \varepsilon_{i}\right)$

where, $\quad\left|\sigma_{s}(r)\right| \varepsilon_{i}=\left[\pi b_{0}^{2}(r) / 4\right]\left[\gamma(r) \varepsilon_{i} / E_{t h}(r)\right]$. Furthermore, by using the result in Equation (11), the 
displacement damage rate per unit volume from Equation (4) is:

$G_{0}(r, t)=n_{a t} \bar{\sigma}_{s}(r)\left[\frac{\bar{\varepsilon}_{i}(r) \gamma(r)}{4 E_{t h}(r)}\right] F_{e x t}(r, t)$

where, $\bar{\sigma}_{s}(r)$ and $\bar{\varepsilon}(r)$ are the average values with respect to the incident particle intensity per unit energy $L_{\text {ext }}\left(t \mid \varepsilon_{i}\right)$ in the energy range of $E_{t h}(r) / \gamma \leq \varepsilon_{i} \leq F_{\text {ext }}(r, t)$ is the integrated external particle intensity for the same energy range and the term in the bracket is the number of Frenkel pairs produced per incident particle.

\section{Point-Defect Diffusion Coefficient}

Even in the absence of particle irradiation, there still exist some thermally activated vacancies at room temperature in a crystal. In this case, the Helmholtz freeenergy function in thermodynamics can be applied by assuming the volume of the crystal is a constant. In the presence of crystal defects, both the entropy $\mathrm{S}$ and the enthalpy $H_{p}$ of a perfect crystal will be changed. A straightforward calculation gives the thermal-equilibrium numbers of vacancies $C_{v}^{e q}$ and interstitials $C_{i}^{e q}$ as follows:

$C_{v, i}^{e q}=\exp \left(\frac{S_{v, i}}{k_{B}}\right) \exp \left(\frac{E_{v, i}}{k_{B} T}\right)$

where $T$ is the temperature, $E_{v}$ is the vacancy formation energy, which is smaller than the interstitial formation energy $E_{i}$ and $S_{v}\left(S_{i}\right)$ is the change in entropy due to vibrational vacancy (interstitial) disorder.

Diffusion of defects is driven by forces other than the concentration gradient of defects, such as stress or strain, electric fields, temperature, etc. The second Fick's law (Murty and Charit, 2013) directly gives rise to the following diffusion equation on the macroscopic scale:

$\frac{\partial C_{v, i}(r, t)}{\partial t}=-\nabla \cdot\left[D_{v, i}(r, t) \nabla C_{v, i}(r, t)\right]$

where, $D_{v}(r, t)=D\left(r, t \mid C_{v}\right)$ and $D_{i}(r, t)=D\left(r, t \mid C_{i}\right)$ are called the diffusion coefficients for vacancy and interstitial atoms, respectively and $c_{v, i}(r, t)=C_{v, \mathrm{i}}(r, t) / V$ is the defect concentrations with $\mathrm{V}$ being the volume of the system considered.

In addition, by assuming a microscopic random walk for the diffusion process, we get the Einstein formula (Guinan et al., 1977):

$D(r)=D_{0}(r) \exp \left[-\frac{E_{a c}(r)}{k_{B} T}\right]=\frac{1}{6} \lambda_{d}^{2}(r) \Gamma(r)$

where, the temperature-independent part, $D_{0}(r)$, is proportional to the Debye frequency $(\sim 10 \mathrm{THz})$ and is independent of defect concentration, $E_{a c}(r)$ is the activation energy for thermal diffusion $\lambda_{d}(r)$ is the diffusion length and $\Gamma(r)$ is the defect jump rate.

For tracer-atom (single radiative atom) diffusion, the random-walk model can not be used. Instead, the diffusion process becomes correlated, described by the Haven coefficient $\mathrm{f}(\mathrm{r})$ and we obtain $D(r)=f(r) \lambda_{d}^{2}(r) \Gamma(r) / 6$ where $\mathrm{f}(\mathrm{r})<1$ depends on the crystal structure and the diffusion mechanism.

The lattice-atom correlated diffusion coefficients $D_{a}^{v, i}(r, t)$ for vacancy and interstitial cases are given by:

$D_{a}^{v, i}(r, t)=f_{v, i}(r) D_{v, i}(r) C_{v, i}(r, t)$

which depend on the defect concentrations in this case, implying a nonlinear diffusion equation. It should be noted that the correlation factors, $f_{v, i}(r)$ and diffusion coefficients, $D_{v, i}(r)$, can be determined by atomic-level simulations (Guinan et al., 1977; Posselt et al., 2008). Equation (16) can be directly applied to defect diffusion under irradiation, as long as the defect concentrations are known.

Mesoscopic-Scale Rate Theory for Slow Defect Migration and Interaction (Defect Reaction and Migration: $t>10 \mathrm{~ns}$ )

The formation, growth and dissolution of defect clusters such as voids, dislocation loops, etc., depend on the diffusion of point defects and their reaction with these defect clusters (Bullough et al., 1979; Colubov et al., 2000). At the same time, they also depend on the concentration of point defects in the crystal. Since particle irradiation greatly raises the defect concentration above its thermal-equilibrium value, the diffusion coefficient can be enhanced. It can also be enhanced by the creation of new defect species.

\section{Point-Defect Diffusion Equation}

By introducing the local coupling rates $\mathfrak{R}(r, t), \Gamma_{i s}(r, t)$ and $\Gamma_{v s}(r, t)$ for vacancy-interstitial recombination, interstitial-sink and vacancy-sink reaction rates, we can write down the following two nonlinear rate-based diffusion equations for binary crystals (Wiedersich et al., 1979):

$$
\begin{aligned}
& \frac{\partial_{C_{v}}(r, t)}{\partial t}=\nabla \cdot\left[D\left(r, t \mid c_{v}\right) \nabla_{C_{v}}(r, t)\right]-\nabla \cdot J_{v}\left(r, t \mid c_{v}\right) \\
& +G_{0}(r, t)-R(r, t) c_{i}(r, t)\left[c_{v}(r, t)-c_{v}^{e q}(r)\right] \\
& -\Gamma_{v s}(r, t) c_{s}(r, t)\left[c_{v}(r, t)-c_{v}^{e q}(r)\right] \\
& \frac{\partial_{c i}(r, t)}{\partial t}=\nabla \cdot\left[D\left(r, t \mid c_{i}\right) \nabla_{c_{i}}(r, t)\right]-\nabla \cdot J_{i}\left(r, t \mid c_{i}\right) \\
& +G_{0}(r, t)-R(r, t) c_{i}(r, t)\left[c_{v}(r, t)-c_{v}^{e q}(r)\right] \\
& -\Gamma_{i s}(r, t) c_{s}(r, t)\left[c_{i}(r, t)\right.
\end{aligned}
$$


where, we have neglected correlated diffusions and defect-defect interactions. Moreover, $J_{v}\left(r, t \mid c_{v}\right)$ and $J_{i}\left(r, t \mid c_{i}\right)$ in Equations (17) and (18) are the particle currents for vacancies and interstitials and the master equation for determining the local sink concentration $c_{s}(r, \mathrm{i})$ will be given later.

For simplicity, we consider a homogeneous system with volume $\mathrm{V}$ in the absence of vacancy and interstitial currents and assume all the rates are independent of time. We further neglect the small thermal-equilibrium vacancy concentration and write the defect generation rate as $G_{0}=G_{0} \mathrm{~V}$. For this model system, we find that the evolution of $c_{v}(t)$ and $c_{i}(t)$ depends on the temperature and $c_{s}$ and can be characterized in several regimes separated by different time scales $\tau$, including initial buildup without reaction, dominant vacancy-interstitial mutual recombination and final vacancy and interstitial annihilation by sinks. Physically, it is easy to understand that the initial buildup and recombination regimes correspond to the 'ultra-fast' atomic-scale modeling, while the interstitial and vacancy annihilation regimes are associated with the 'slow' mesoscopic-scale modeling which may be simulated using the so-called rate theory (Singh and Zinkle, 1993) or phase field model (Li et al., 2010).

\section{Recombination and Sink Annihilation Rates}

In general, the reaction rate between species $A$ and B can be expressed as $\Gamma_{A B} c_{A} c_{B}$ where $c_{A}$ and $c_{B}$ are the concentrations (particles $\left./ \mathrm{cm}^{3}\right)$ and $\Gamma_{A B}\left(\mathrm{~cm}^{3} / \mathrm{s}\right)$ is the rate constant.

As an example, the recombination rate constant $\mathfrak{R}(r, t)$ in Equation (17) and (18) for vacancies and interstitials takes the form of (Waite, 1957):

$$
\mathfrak{R}(r, t)=\frac{z_{i v}(r) \Omega(r) D_{i}(r, t)}{a_{0}^{2}(r)}
$$

where, $z_{i v}(r)$ (an integer) is the bias factor, depending on the crystal structure and species, $\Omega(r)$ is the atomic volume, $a_{0}(r)$ is the lattice constant and $D_{i}(r, t)$ is the mobile interstitial diffusion coefficient.

In a similar way, the interstitial-sink $\Gamma_{i s}(r, t)$ or the vacancy-sink $\Gamma_{v s}(r, t)$ annihilation rate constants in Equations (17) and (18) are given by $\Gamma_{a s}(r, t) c_{a}(r, t) c_{s}(r$, $t)=k_{a s}^{2}(r, t) c_{a}(r, t) D_{a}(r, t)$, where $\alpha$ corresponds to mobile defect species and $\kappa_{\alpha s}(\mathrm{r}, \mathrm{t})\left(\mathrm{cm}^{-2}\right)$ represents the sink strength given by (Doan and Martin, 2003):

$$
\kappa_{a s}^{2}(r, t)=\frac{\Gamma_{a s}(r, t) c_{s}(r, t)}{D_{a}(r, t)}
$$

The sink strength measures the affinity of a sink for defects, which is independent of defect properties and $\kappa_{a s}^{-1}(r, t)$ corresponds to the mean distance for a traveling defect in the crystal before it is trapped by sinks.

\section{Point-Defect Interaction Rates}

In the absence of the macroscopic-scale gradient of defect concentration, the reaction between defects and sinks is reaction-rate-controlled. According to Equation (19), the defect-void interaction (Brailsford et al., 1976) can be described by the rate constants $\Gamma_{\{i, v\}}(r, t)$ by:

$$
\begin{aligned}
& \Gamma_{\{i, v\} v}(r, t)=\sum_{n=2}^{\infty} \frac{4 \pi R^{2}(r, t \mid n) D_{i, v}(r, t)}{a_{0}(r)} \\
& =\sum_{n=2}^{\infty} \frac{\kappa_{V}^{2}(r, t \mid n) D_{i, v}(r, t)}{c_{V}(r, t \mid n)}
\end{aligned}
$$

where, $\quad z_{\{i, v\} V}=4 \pi R^{2} / a_{0}^{2}, \Omega \sim a_{0}^{3}, R(r, t \mid n) \quad$ represents the radius of a void sphere involving $\mathrm{n}$ vacancies. The void strength is given by $\kappa_{V}^{2}(r, t \mid n)=4 \pi R^{2}(r, t \mid n) c_{V}(r, t \mid n) / a_{0}(r)$ where $c_{\nu}(r, t \mid n)$ is the concentration of voids containing $\mathrm{n}$ vacancies in the crystal.

Similarly, for the defect-dislocation interaction, we have the rate constants $\Gamma_{\{i, v\} d}(r, t)$ (Heald and Speight, 1975) (in units of $\mathrm{cm}^{2} / \mathrm{s}$ ) given by:

$\Gamma_{\{i, v\} d}(r, t)=z_{\{i, v\} d}(r) D_{i, v}(r, t)=\frac{\kappa_{\{i, v\} d}^{2}(r, t) D_{i, v}(r, t)}{\rho_{d}(r, t)}$

where, we replace $\Omega$ in Equation (19) by an atomic area $\left(\sim a_{0}^{2}\right), z_{i d}(r) \neq z_{v d}(r)$ and $\rho_{\mathrm{dr}, \mathrm{t}}(\mathrm{r}, \mathrm{t})$ is the dislocation areal density.

Reactions driven by defect concentration gradients are diffusion limited instead of reaction-rate limited as discussed above. In this case, we have to solve the diffusion term $\nabla \cdot\left[D_{i, v}(r, t) \nabla_{c i, v}(r, t)\right]$ with the generation term $\mathrm{G}_{0}(\mathrm{r}, \mathrm{t})$ for spherical (voids) or cylindrical (dislocation lines) coordinates. In the case of dislocation loops, the sink strength is more complicated and increases with increasing loop size (Bullough et al., 1979).

For the defect-void interaction, we get $\Gamma_{\{i, v\} V}(r, t)=\sum_{n=2}^{\infty} \Gamma_{\{i, v\} V}(r, t \mid n), \Gamma_{\{i, v\} V}(r, t \mid n)=4 \pi R(r, t \mid n) D_{i, v}(r, t)$ and $\kappa_{V}^{2}(r, t \mid n) 4 \pi R(r, t \mid n) c_{V}(r, t \mid n)$. For the defect-dislocation line interaction, on the other hand, we have $\Gamma_{\{i, v\} d}(r, t)=2 \pi D_{i, v}(r, t) / \operatorname{In}\left[R_{0} / R_{\{i, v\} d}(r, t)\right]$ and $\kappa_{\{i, v\} d}^{2}(r, t) / \operatorname{In}\left[R_{0} / R_{\{i, v\} d}(r, t)\right]$, where $\mathrm{R}_{0}$ is the absorption radius of a sink, $\mathrm{R}_{\mathrm{id}}(\mathrm{r}, \mathrm{t})$ and $\mathrm{R}_{\mathrm{vd}}(\mathrm{r}, \mathrm{t})$ are the sink capture radii for interstitials and vacancies, respectively with $R_{i d} \gg R_{v d}$.

In the presence of Grain Boundaries (GBs), we obtain the grain-boundary sink strength: 


$$
\begin{aligned}
& \kappa_{\{i, v\} g b}^{2}(r, t)=4 \pi R_{g b}(r) c_{g b}(r) \\
& \times\left\{\begin{array}{c}
\kappa_{i, v}(r, t) R_{g b}(r) \cosh \left[\kappa_{i, v}(r, t) R_{g b}(r)\right] \\
\frac{-\sinh \left[\kappa_{i, v}(r, t) R_{g b}(r)\right]}{\sinh \left[\kappa_{i, v}(r, t) R_{g b}(r)\right]-\left[\kappa_{i, v}(r, t) R_{g b}(r)\right.}
\end{array}\right\}
\end{aligned}
$$

where, $R_{g b}(r)$ is the radius of a spherical grain, $c_{g b}(r)$ is the grain concentration and $\kappa_{i, v}(r, t)$ is the sink strength for the grain interior for interstitlals or vacancies due to dislocations and voids. Moreover, its rate constant is $\Gamma_{\{i, v\} g b}(r, t)=\kappa_{\{i, v\} g b}^{2}(r, t) D_{i, v}(r, t) / c_{g b}(r)$. More recently, atomic-level simulations have been employed to study the sink strength of grain boundaries (Tschopp et al., 2012; Jiang et al., 2014). These simulations revealed that the sink strength can exceed the theoretical strength of GBs due to the effect of GB stress fields.

\section{Radiation-Induced Segregation}

For a binary A-B alloy (or donor and acceptor randomly-doped semiconductors), in the absence of sinks, the diffusion equations for vacancies, interstitials and atoms $\mathrm{A}$ and B are (Wiedersich et al., 1979):

$$
\begin{aligned}
& \frac{\partial c_{v}(r, t)}{\partial t}=-\nabla \cdot J_{v}(r, t)+G_{0}(r, t)-\mathfrak{R}(r, t) c_{i}(r, t) c_{v}(r, t) \\
& =\nabla\left\{-\left[d_{A v}(r)-d_{B v}(r)\right] X(r, t) \Omega(r) c_{v}(r, t)\right. \\
& \left.\nabla c_{A}(r, t)+D_{v}(r, t) \nabla c_{v}(r, t)\right\} \\
& +G_{0}(r, t)-\mathfrak{R}(r, t) c_{i}(r, t) c_{v}(r, t) \\
& \frac{\partial c_{v}(r, t)}{\partial t}=-\nabla \cdot J_{v}(r, t)+G_{0}(r, t)-\mathfrak{R}(r, t) c_{i}(r, t) c_{v}(r, t) \\
& =\nabla\left\{-\left[d_{A i}(r)-d_{B i}(r)\right] X(r, t) \Omega(r)\right. \\
& \left.c_{i}(r, t) \nabla c_{A}(r, t)+D_{i}(r, t) \nabla c_{i}(r, t)\right\} \\
& +G_{0}(r, t)-\mathfrak{R}(r, t) c_{i}(r, t) c_{v}(r, t) \\
& \frac{\partial c_{v}(r, t)}{\partial t}=-\nabla \cdot J_{A}(r, t)+\nabla\left\{D_{A}(r) X(r, t) \nabla c_{A}(r, t)\right. \\
& \left.+\Omega(r) c_{A}(r, t)\left[d_{A i}(r) \nabla c_{i}(r, t)-D_{A v}(r) \nabla c_{v}(r, t)\right]\right\}
\end{aligned}
$$

where, $\quad d_{\{A, B\}\{i, v\}}(r)=\lambda_{i, v}^{2}(r) z_{i, v}(r) \omega_{\{A, B\}\{i, v\}}(r) \quad$ are the diffusivity coefficients and the dimensionless $X(r, t)$ is the thermodynamic factor connecting the chemicalpotential gradient to the concentration gradient (or built-in electric field at junctions). In addition, we have $c_{B}(r, t)=\tilde{\Omega}^{l}(r)-c_{A}(r, t)$ when small defect concentrations are neglected.

By requiring $J_{A}=J_{B}=0$ and $J_{i}=J_{v}$ for steady state and neglecting $G_{0}(r, t)$ and $\mathfrak{R}(r, t)$ in Equation (24)(26), we get:

$$
\begin{aligned}
& \nabla c_{A}=-\nabla c_{B}=\frac{N_{A} N_{B} d_{B i} d_{A i}}{X\left(d_{B i} N_{B} D_{A}+d_{A i} N_{A} D_{B}\right)} \\
& \left(\frac{D_{A v}}{d_{B v}}-\frac{d_{A i}}{d_{B i}}\right) \nabla c_{V}
\end{aligned}
$$

where, $N_{A, B}=c_{A, B} \Omega$ and the direction of $\nabla c_{A}$ can be either parallel or anti-parallel to $\nabla c_{V}$. Additionally, the undersized (oversized) solutes bounded to interstitials will be concentrated (depleted) around sinks to create a concentration gradient after their redistribution.

On the other hand, the oversized or undersized solutes with respect to the lattice atoms can act as traps for vacancies and interstitials, including release of defects from traps, recombination with trapped defects, trapping of free point defects and loss to internal sinks. This is further supplemented by three rate equations for trap and trapped defect concentrations.

\section{Sink Dynamics}

The growth of dislocation loops and spherical voids is determined by solving the point-defect balance equations without diffusion terms. Since the defect concentration is still changing with time due to the timedependent radiation source (or defect production rate), only quasi-steady state can be defined for short periods of time. Physically, the quasi-steady state is related to the fact that the change in sink strength due to microstructure evolution is slow compared to the response time of the defect population.

\section{Planar Biased Dislocation-Loop Growth}

By defining the dislocation line direction $s$ and the Burgers vector $b$ for edge $(b \perp s)$ or skew $(b \| s)$ dislocations, the Peach-Koehler equation (Weertman, 1965) gives us the force f per length as:

$$
f=b^{T} \cdot(\underline{\sigma} \times s)
$$

where, $\underline{\sigma}$ is the stress tensor. The force $\mathrm{f}$ along the $\mathrm{b}$ direction is the glide force, while $\mathrm{f}$ perpendicular to both $\mathrm{s}$ and $\mathrm{b}$ directions is called the climb force. The PeachKoehler equation can be used for calculating the interaction between dislocations, where $\mathrm{b}$ and $\mathrm{s}$ are assigned to the target dislocation while $\underline{\sigma}$ is for the source dislocation. For an edge dislocation, we have five non-zero stress tensor elements $\sigma_{x x}, \sigma_{y y}, \sigma_{z z}$ and $\sigma_{x y}=\sigma_{y c}$, while for a skew dislocation, we only have four non-zero stress tensor elements $\sigma_{y x}=\sigma_{z y}$ and $\sigma_{x z}=\sigma_{z x}$.

Besides the dislocation lines, there also exists Frank loops. For example, the close-packed fcc lattice follows the stacking sequence $\mathrm{ABCABCABC} \mathrm{...,} \mathrm{where} \mathrm{A}, \mathrm{B}$ and $C$ correspond to different planes of atoms. It can be modified to $\mathrm{ABCAB} / \mathrm{ABC}$..., where "/" denotes the 
intrinsic single fault or missing plane of atoms. It can also be modified to $\mathrm{ABCAB} / \mathrm{A} / \mathrm{CABC} \ldots$, where a plane of atoms or the extrinsic double fault is inserted.

Interstitial condensation can occur around the edges of the depleted zone. A cluster of point defects can be a line, a disc or a void. The formation of perfect or faulted loops of interstitials competes with the formation of voids of vacancies, which are also affected by the irradiation temperature.

The nucleation of loops is a clustering process that results in a critical size embryo for further growth. As an example, by denoting the number of clusters consisting of $j$ vacancies as $\rho_{v}(j)$, the master equations for $\rho_{v}(j)$ are:

$$
\begin{aligned}
& \frac{\partial \rho_{v}(j)}{\partial t}=G_{0}(j)-\sum_{n=1}^{\infty}\left[\beta_{v_{n}}(j)+\beta i_{n}(j)\right] \\
& \rho v(j)-\sum_{n=1}^{j} a_{v_{n}}(j) \rho v(j) \\
& +j-1 \sum_{n=1} \beta v_{n}(j-n) \rho_{v}(j-n)+\sum_{n=1}^{\infty}\left[\beta_{v_{n}}(j-n) \rho v(j+n)+\right. \\
& \sum_{n=1}^{\infty}\left[\beta_{v_{n}}(j+n) \rho v(j+n)\right.
\end{aligned}
$$

where, $\beta_{v n}$ and $\beta_{i n}$ are the capture rates of migrating vacancy (v) or interstitial (i) clusters of size $\mathrm{n}$ by a cluster of size $\mathrm{j}$ and $a_{v}(j)$ is the emission rate for the new vacancy cluster of size $\mathrm{n}$ by a cluster of size $\mathrm{j}$. In Equation (29), the first term is the direct production of a cluster of size $\mathrm{j}$, while the second term is the loss of clusters from size $\mathrm{j}$ due to absorption of a cluster of size $n$. The third term is the loss of a cluster of size $\mathrm{j}$ due to emission of a cluster of size n. The fourth and fifth terms in Equation (29) are the addition of clusters to the cluster of size $\mathrm{j}$ due to absorption of vacancy clusters by a smaller cluster and absorption of interstitial clusters by a larger cluster and the last term is the addition of clusters to the cluster of size $j$ due to loss of vacancy clusters by a larger cluster.

Since the dominant contribution for cluster reactions is with point defects (i.e., cluster of size $\mathrm{j}=1$ ), for both vacancies and interstitials, Equation (29) with $j \geq 2$ can be simplified to:

$$
\begin{aligned}
& \frac{\partial \rho(j ; t)}{\partial t}=G_{0}(j ; t)+\beta(j-1, j) \\
& \rho(j-1 ; t)+a(j+1) \rho(j+1 ; t) \\
& -\beta(j, j+1)+a(j, j-1) \rho(j ; t)
\end{aligned}
$$

If the cluster size index $\mathrm{j}$ can be treated as a continuous variable $\xi$, Equation (30) reduces to a Fokker-Planck equation (Semenov and Woo, 2002).

According to Equation (30), for the dislocation loop growth, we find the evolution of the number density $\rho_{i l}(j$, $t$ ) for the interstitial loop of size $\mathrm{j}$ satisfies:

$$
\begin{aligned}
& \frac{\partial \rho(j ; t)}{\partial t}=\left.G_{0}(j ; t)\right|_{j \geq 4}+\left[\beta_{v}(j+1)\right. \\
& \left.+a_{i}(j-1)\right] \rho_{i l}(j+1 ; t)+\beta_{i}(j-1) \rho_{i l}(j-1 ; t) \\
& -\beta_{v}(j)+\beta_{i}(j) a_{i}(j) \rho_{i l}(j ; t)
\end{aligned}
$$

Where:

$$
\beta_{i, v}(j)=2 \pi r(j) z_{c}(j) D_{i, v}(r, t) C_{i, v}(r, t)
$$

$a_{i, v}(j)=2 \pi r(j) z_{c}(j) \frac{D_{i, v}(r, t)}{\Omega} \exp \left[-\frac{E_{b,\{i, v\}}(j)}{k_{B} T}\right]$

$r(j)$ and $z_{c}$ are the radius and bias factor of an interstitial loop of size $j$ and $E_{b}(j)$ is the binding energy for a cluster of $\mathrm{j}$ defects.

The saturation of the dislocation density $\rho_{d}(t)$ in quasi-steady state was found experimentally to be due to a recovery process at high temperatures, (Was, 2007) given by:

$$
\frac{d \rho_{d}(t)}{d t}=A_{2} \rho_{d}(t)-A_{1} \rho_{d}^{2}(t)
$$

where, $A_{2}$ and $A_{1}$ are constants. This gives the steadystate solution $\rho_{d}^{s}=A_{2} / A_{1}$ and the time-dependent solution is found to be:

$$
\frac{\rho_{d}(t)}{\rho_{d}^{s}}=\left[\frac{1-e^{-x}+\sqrt{\rho_{d}^{0} / \rho_{d}^{s}}\left(1+e^{-x}\right)}{1-e^{-x}+\sqrt{\rho_{d}^{0} / \rho_{d}^{s}}}\left(1+e^{-x}\right)\right]^{2}
$$

where, $\rho_{d}^{0}$ is the initial value and $x(t)=A_{1} \sqrt{\rho_{d}^{s}} t$. In addition, a phase field model has been developed to describe the growth kinetics of interstitial loops in irradiated materials during aging. The diffusion of vacancies and interstitials and the elastic interaction between interstitial loops and point defects are accounted for in this model. The effects of interstitial concentration, chemical potential and elastic interaction on the growth kinetics and stability of interstitial loops are investigated in two and three dimensions. The elastic interaction enhances the growth kinetics of interstitial loops. The elastic interaction also affects the stability of a small interstitial loop adjacent to a larger loop. The linear growth rates for interstitial loops predicted is in agreement with the previous theoretical predictions and experimental observations (Hu et al., 2012).

\section{Spherical Neutral Void Growth}

Not all the defects generated by radiation-induced atom displacement are point defects. Some of the defects form clusters and the vacancy clusters may further grow to form voids. For a small number of vacancies, the 
spherical void is favorable, while for a large number of vacancies, the planar loop is a more stable configuration.

The dynamics for void growth is very similar to that for dislocation-loop growth. The net absorption rate of vacancies by a spherical void is the difference of absorption rates of vacancies and interstitials, i.e., $A_{n e t}^{V}=4 \pi R_{V}\left\{D_{v}\left[V_{v}-C_{v}(R)\right]-D_{i} C_{i}\right\} . \quad$ Therefore, the equation for the growth of a spherical void of radius $R_{\nu}(t)$ (or volume) in quasi-steady state is:

$$
\begin{aligned}
& \frac{d R_{V}(t)}{d t}=\frac{\Omega}{R_{V}(t)}\left\{D _ { v } ( r , t ) \left[c_{v}(r, t)\right.\right. \\
& \left.\left.-c_{v}\left(R_{V}\right)\right]-D_{i}(r, t) c_{i}(r, t)\right\}
\end{aligned}
$$

where, $c_{v}\left(R_{V}\right)$ is the vacancy concentration at the void surface.

From the balance equation, we get the concentrations of point vacancies and interstitials as follows:

$$
c_{i, v}(r, t)=\frac{D_{i, v}(r, t) k_{i, v}^{2}(r, t)}{2 R(r, t)}[\sqrt{\eta+1}-1]
$$

where,

$k_{i, v}^{2}(r, t)=z_{i, v}(r) \rho_{d}(r, t)+4 \pi R_{V}(r, t) c_{V}(r, t), \eta=4 R G_{0} / D_{i} D_{v} k_{v}^{2} k_{i}^{2}$ . Inserting Equation (37) into Equation (36), we obtain:

$\frac{d R_{V}(t)}{d t}=\frac{d R_{0}(t)}{d t}\left\{\frac{2}{\eta}[\sqrt{\eta+1}-1]\right\}-\frac{d R_{t h}(t)}{d t}$

where, $d R_{0}(t) / d t$ is proportional to $\left(z_{i}-z_{v}\right) G_{0}$ and independent of temperature, while the second negative term represents the thermal emission of defects from sinks and strongly depends on temperature (proportional to $D_{v} C_{v}^{e q}$ ).

Neglecting the thermal emission in Equation (38), we get from Equation (36):

$$
\frac{d R_{V}}{d t} \approx \frac{\Omega D_{v} D_{i}}{2 R_{V} R}\left[1+\sqrt{\frac{4 R G_{0}}{D_{i}, D_{v} k_{v}^{2} k_{i}^{2}}}-1\right] \rho d\left(z_{i}^{d} z_{v}-z_{v}^{d} z_{i}\right)
$$

where, the sign of the bias of dislocation $\left(z_{i}^{d} z_{v}-z_{v}^{d} z_{i}\right)$ for vacancies and interstitials determines the occurrence of either growth $\left(d R_{V} / d t>0\right)$ or shrinkage $\left(d R_{V} / d t<0\right)$. It should be noted that void growth in materials under radiation has been recently studied by the phase field model, (Li et al., 2010) which provides important insights into the growth kinetics of voids. The model takes into account the generation of vacancies and interstitials associated with the irradiation damage, the recombination between vacancies and interstitials, defect diffusion and defect sinks. The results demonstrate that the temperature gradient causes void migration and defect fluxes, i.e., the Soret effect, which affects void stability and growth kinetics. It is found that the effect of defect concentration, generation rate and recombination rate on void mobility for migration is minor although they strongly influence the void growth kinetics.

\section{Radiation Degradation of Electronic Devices}

Let us consider a commonly used layered-structure material, (Huang and Zhou, 1988) as shown in Fig. 3. Each material layer is characterized by the radiation parameters $G_{j}, \mathfrak{R}_{j}, D_{j}$ and $\Gamma_{j}$ with $\mathrm{j}=1,2,3,4$ for generation and recombination rates, diffusion coefficient and bulk-sink annihilation, which will be employed to model the dynamics from an ultra-fast atomic-scale up to 100 ns. The calculated non-steady state defect distribution in each layer will be used for initial conditions in a slow mesoscopic-scale diffusion and annihilation model in order to calculate the steady-state spatial distribution of defects in the whole layered structure. In modeling the mesoscopic-scale, the interface-sink strengths $k_{i}^{2}$ with $\mathrm{i}=1,2,3$ will also be considered. Once the steadystate distribution of point defects, denoted as $\rho_{d}(z)$, is obtained for the whole layered structure, they will be fed into the follow-up calculations for radiation degradation in electronic devices, as described below.

The band structure of a crystal largely determines the properties of electrons, (Gumbs and Huang, 2013) such as effective mass, bandgap energy, density of states, plasma frequency and absorption coefficient. These electron properties are a result of the unique crystal potential from all lattice atoms, instead of properties of an individual lattice atom. On the other hand, the radiation-induced displacements of lattice atoms are determined not only by the intrinsic properties, such as mass of the atoms, but also by the extrinsic conditions, such as kinetic energy of incident particles and lattice temperature.

\section{Steady-State Defect Distributions}

For the reaction rate control system shown in Fig. 3, by generalizing Equations (17) and (18), we write down the diffusion equations for point vacancies and interstitials as:

$$
\begin{aligned}
& \frac{\partial c_{v}^{j}(z, t)}{\partial t}=D_{v}^{j} \frac{\partial^{2} c_{v}^{j}(z, t)}{\partial z^{2}}+G_{0}^{j}(t) \\
& -\left[\frac{z_{i v}^{j} \Omega_{j} D_{i}^{j}}{\left(a_{0}^{j}\right)^{2}}\right] c_{i}^{j}(z, t)\left[c_{v}^{j}(z, t)-c_{v}^{e q}(j)\right] \\
& -\sum_{n=2}^{\infty}\left\{\frac{4 \pi\left[R_{V}^{j}(t \mid n)^{2} D_{v}^{j}\right]}{a_{0}^{j}}\right\} c_{V}^{j}(z, t \mid n)\left[c_{v}^{j}(z, t)-c_{v}^{e q}(j)\right] \\
& -\sum_{n=2}^{\infty}\left[z_{v d}^{j+1} \rho_{d}^{j+1}(t \mid n) D_{v}^{j+1}\right]\left[c_{v}^{j+1}(z, t)-c_{v}^{e q}(j+1)\right] a_{0}^{j+1} \delta\left(z-z_{j+1}\right)
\end{aligned}
$$




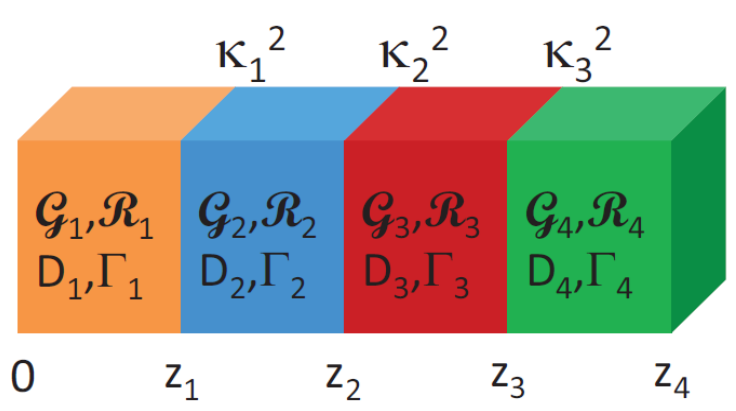

Fig. 3. Layered-structure material with radiation parameters $\mathrm{G}_{\mathrm{j}}$, $R_{j}, D_{j}$ and $\Gamma_{j}(j=1,2,3,4)$ for generation, recombination, diffusion coefficient and bulk-sink annihilation, respectively. In addition, $k_{i}^{2}$ for $\mathrm{i}=1,2,3$ represents the interface-sink strengths. Particles are incident from the front surface at $\mathrm{z}=0$ and exit from the back surface at $\mathrm{z}=\mathrm{z}_{4}$

$$
\begin{aligned}
& -\sum_{n=2}^{\infty}\left[z_{v d}^{j} \rho_{d}^{j}(t \mid n) D_{v}^{j}\right]\left[c_{v}^{j}(z, t)-c_{v}^{e q}(j) a_{0}^{j} \delta\left(z-z_{j}\right)\right], \\
& \frac{\partial c_{v}^{j}(z, t)}{\partial t}=D_{i}^{j} \frac{\partial^{2} c_{i}^{j}(z, t)}{\partial z^{2}}+G_{0}^{j}(t) \\
& -\left[\frac{z_{i v}^{j} \Omega_{j} D_{i}^{j}}{\left(a_{0}^{j}\right)^{2}}\right] c_{i}^{j}(z, t)\left[c_{v}^{j}(z, t)-c_{v}^{e q}(j)\right] \\
& -\sum_{n=2}^{\infty}\left\{\frac{4 \pi\left[R_{V}^{j}(t \mid n)\right]^{2}}{a_{0}^{j}}\right\} c_{v}^{j+1}(t \mid n) c_{i}^{j}(z, t) \\
& -\sum_{n=2}^{\infty}\left[z_{i d}^{j+1} \rho_{d}^{j+1}(t \mid n) D_{i}^{j+1}\right] c_{i}^{j+1}(z, t) a_{0}^{j+1} \delta\left(z-z_{j+1}\right) \\
& -\sum_{n=2}^{\infty}\left[z_{v d}^{j} \rho_{d}^{j}(t \mid n) D_{i}^{j}\right] c_{i}^{j}(z, t) a_{0}^{j} \delta\left(z-z_{j}\right)
\end{aligned}
$$

where, the integer $\mathrm{j}$ is the layer index, $z_{j-1}$ and $z_{j}$ represent the left and right interface positions of the jth layer, respectively. In Equations (40) and (41), we used the facts that $\Gamma_{i, v}=z_{i, v} \Omega D_{i, v} / a_{0}^{2}, \kappa_{V}^{2}=4 \pi R_{V}^{2} c_{V} / a_{0}$ and $\kappa_{\{i, v\} d}^{2}=z_{\{i, v\}} d \rho d$ for a reaction rate control system.

The diffusion coefficients $D_{i, v}^{j}$ for point vacancies and interstitials are calculated as:

$$
\begin{aligned}
& D_{i, v}^{j}(T)=f_{i, v}^{j} \eta_{j}\left(a_{0}^{j}\right)^{2} \exp \left[\frac{\Delta S_{m}^{i, v}(j)}{k_{B}}\right] \\
& \exp \left[\frac{E_{m}^{i, v}(j)}{k_{B} T}\right]=f_{i, v}^{j} D_{0} \exp \left[\frac{E S_{m}^{i, v}(j)}{k_{B} T}\right]
\end{aligned}
$$

where, $f_{i, v}^{j}<1$ is the diffusion correlation factor, $\eta_{j}$ is the structural factor relating to the jump distance and number of nearest neighbors, $v_{j}$ is the jump frequency on the order of the Debye frequency, $\Delta S_{m}^{i, v}(j)$ is the change in entropy due to vibrational defect disorder and $E_{m}^{i, v}(j)$ is the point-defect migration energy.

The radius $R_{V}^{j}(t \mid n)$ of the spherical void of size $\mathrm{n}$ (in unit of lattice constant) introduced in Equations (40) and (41) is determined from the following void growth equation:

$\frac{d R_{V}^{j}(t \mid n)}{d t}=\frac{\Omega_{j}}{R_{V}^{j}(t \mid n)}\left\{D_{v}^{j}\left[c_{v}^{j}(z, t)-c_{v}^{j}\left(t \mid R_{V}\right)\right]-D_{i}^{j} c_{i}^{j}(z, t)\right\}$

where, $c_{v}^{j}\left(t \mid R_{V}\right)$ is the vacancy concentration at the void surface and is given by:

$c_{v}^{j}\left(t R_{V}\right)=\frac{c_{v}^{0}(j) \Omega_{j} z_{v}^{j} \rho_{d}^{j}(t)\left[2 \gamma_{j} / R_{V}^{j}(t \mid n)-\sigma_{j}\right]}{k_{B} T\left[z_{v}^{j} \rho_{d}^{j}(t)+4 \pi R_{V}^{j}(t \mid n) c_{V}^{j}(t \mid n)\right]}$

$\gamma_{j}$ is the surface tension of the void, $\sigma_{j}$ is the hydrostatic stress applied to the void and $c_{v}^{0}(j)$ is the thermalequilibrium vacancy concentration, given by:

$$
\begin{aligned}
& c_{v}^{0}(j)=\frac{1}{\Omega_{j}} \exp \left[\frac{\Delta S_{f}^{v}(j)}{k_{B}}\right] \\
& \exp \left[-\frac{E_{f}^{v}(j)}{k_{B} T}\right]=n_{0}(j) \exp \left[-\frac{E_{f}^{v}(j)}{k_{B} T}\right]
\end{aligned}
$$

$\Delta S_{f}^{j}$ is the change of entropy for the formation of a point vacancy and $E_{f}^{j}$ is the point vacancy formation energy.

By using a continuous variable, the void concentration $c_{V}^{j}(z, t \mid n)($ with $n \geq 2)$ introduced in Equations (40) and (41) can be obtained by solving the Fokker-Planck equation in the size space below (with $\xi=n$ ):

$$
\begin{aligned}
& \frac{\partial c_{V}^{j}(z, t \mid \xi)}{\partial t}=G_{0}^{j}(t \mid \xi)-\frac{\partial}{\partial \xi} \\
& \left\{F_{j}(z, t \mid \xi) c_{V}^{j}(z, t \mid \xi)-\frac{\partial}{\partial \xi}\left[D_{j}(z, t \mid \xi) c_{V}^{j}(z, t \mid \xi)\right]\right\}
\end{aligned}
$$

where, $\quad F_{j}(z, t \mid \xi)=z_{v}^{j} D_{v}^{j} c_{v}^{j}(z, t)-z_{i}^{j} D_{i}^{j} c_{i}^{j}(z, t)$ is the drift term, $\quad D_{j}(z, t \mid \xi)=\left[z_{v}^{j} D_{v}^{j} c_{v}^{j}(z, t)+z_{i}^{j} D_{i}^{j} c_{i}^{j}(z, t)\right] / 2$ is the positive diffusion term and $G_{0}^{j}(t \mid \xi)$ is the cluster production rate per volume (with $\zeta \geq 2$ ).

Finally, the dislocation-loop density $\rho_{d}^{j}(t \mid n)$ introduced in Equations (40) and (41) can be found from (with $n \geq 4$ ):

$$
\begin{aligned}
& \frac{\partial \rho_{d}^{j}(t \mid n)}{\partial t}=G_{0}\left(z_{j}, t \mid n\right)+\left[\begin{array}{l}
\beta_{v}^{j}(t \mid n+1) \\
+a_{i}^{j}\left(z_{j}, t \mid n+1\right) \rho_{d}^{j}(t \mid n+1)
\end{array}\right] \\
& +\beta_{i}^{j}\left(z_{j}, t \mid n-1\right) \rho_{d}^{j}\left(t \mid n-1-\left[\beta_{v}^{j}\left(z_{j}, t \mid n\right)\right.\right. \\
& \left.+\beta_{i}\left(z_{j}, t \mid n\right)+a_{i}\left(z_{j}, t \mid n\right) \rho_{d}^{j}(t \mid n]\right)
\end{aligned}
$$


where, $G_{0}\left(z_{j}, t \mid n\right)$ is the production rate per unit volume for the interstitial dislocation loop of length $n$, the absorption $\left(\beta_{i, v}\right)$ and emission $\left(\alpha_{i, v}\right)$ rates in Equation (47) are defined by:

$$
\beta_{i, v}^{j}\left(z_{j}, t \mid n\right)=2 \rho \ell_{j}(n) z_{c}^{j}(n) D_{i, v}^{j} c_{i, v}^{j}\left(z_{j}, t\right)
$$

$a_{i, v}^{j}\left(z_{j}, t \mid n\right)=2 \pi \ell_{j}(n) z_{c}^{j}(n)\left[\frac{D_{i, v}^{j}}{\Omega_{j}}\right] \exp \left[-\frac{E_{b,\{i, v\}}^{j}(n)}{k_{B} T}\right]$

$\ell_{j}(n)$ and $z_{c}^{j}(n)$ are the radius and bias factor of an interstitial loop of size $\mathrm{n}$ and $E_{b}^{j}(n)$ is the binding energy for a cluster of $\mathrm{n}$ interstitial atoms.

The initial condition for the diffusion equations will be given by the corresponding calculated results from the atomic-scale model for individual layers. The point defect diffusion occurs mainly around interfaces between two adjacent layers or across the interfaces. The boundary conditions with continuous concentrations of point defects, as well as the jump in their derivatives determined by the dislocation sinks, will be applied at each interface. In addition, the constraints for the zero concentration of point defects as well as the zero derivative of the concentration with respect to $\mathrm{z}$ at the two surfaces of the system will be enforced in our numerical computations.

\section{Point-Defect Electronic States}

To study the defect degradation effect on devices, we need to know not only the concentration and spatial distribution $\rho_{\mathrm{d}}(\mathrm{r})$ of the irradiation-induced defects but also their electronic properties, such as energy level $E_{\mathrm{j}}$, wave function $\psi_{\mathrm{j}}(\mathrm{r})$ and local density of states $D_{d}(r, E)$. Although the semi-classical MD calculation and the reaction-rate theory allow us to obtain the concentration and spatial distribution of defects, we still require Density-Functional Theory (Drabold and Estreicher, 2007; Freysoldt et al., 2014) (DFT) for calculating defect configurations, energy levels, density of states and charge trapping by point defects in crystals.

The main idea of DFT is to reformulate the energy of an atomic system as a functional of the ground state electron density function $\rho_{0}(r)$ instead of individual electron wave functions. The proof of existence of such a functional relies on a one-to-one correspondence between the external potential $V_{e x t}\left(\left\{R_{\ell}\right\},\left\{r_{m}\right\}\right)$ and $\rho_{0}(r)$, where $\left\{R_{\ell}\right\}$ and $\left\{r_{m}\right\}$ label all the lattice atoms and electrons, respectively. The mapping of $V_{\text {ext }}\left(\left\{R_{\ell}\right\}\right.$, $\left.\left\{r_{m}\right\}\right)$ onto $\rho_{0}(r)$ is obvious. Any Hamiltonian $\hat{H}$ with a given external potential $V_{e x t}\left(\left\{R_{\ell}\right\},\left\{r_{m}\right\}\right)$ has a ground state solution with an N-electron wave function $\varphi_{0}\left(\left\{r_{m}\right\}\right)$, which can be uniquely associated with the electron density function $\rho_{0}(r)$ using:
$\rho_{0}(r)=N f \ldots f\left|\varphi_{0}\left(r_{1}, r_{2}, \ldots, r_{N}\right)\right|^{2} \delta\left(r-r_{1}\right) d^{3} r_{1} d^{3} r_{2} \ldots d^{3} r_{N}$

Due to the resulting one-to-one correspondence between $V_{e x t}\left(\left\{R_{\ell}\right\},\left\{r_{m}\right\}\right)$ and $\rho_{0}(r)$, the energy $E_{i}$ of the atomic system can be expressed as a functional of the electron density $\rho_{0}(r)$. The many-electron wave function of $\varphi\left(r_{1}, r_{2}, \ldots, r_{N}\right)$ depends on the 'combination' of all spatial electron coordinates. Unfortunately, such an approach would by far exceed any computational capabilities. However, this problem can be overcome by using the Kohn-Sham (KS) ansatz, (Kohn and Sham, $1965)$ in which the fully-interacting system is replaced by a non-interacting one. This approach corresponds to a mean-field approach, where the many-electron wave function is decomposed into a product of $\mathrm{N}$ singleelectron orbitals $\phi_{i}(r)$ (i.e., Slater determinant). This simplification leads to a neglect of an energy contribution termed 'correlations'. As a correction, the functional $E_{x c}[\rho(r)]$ must be introduced as an additional term in the Hamiltonian. Applying the variation principle to the modified Hamiltonian yields a single-particle-like Schrodinger equation, also referred to as the Kohn-Sham equation in DFT. This equation includes an effective potential $V_{\text {eff }}(r)$, which is produced by the Coulomb forces of all other electrons and nuclei and incorporates the exchange and correlation interactions, i.e.,:

$\left[-\frac{h^{2}}{2 m_{e}} \nabla^{2}+V_{e f f}(r)\right] \phi_{i}^{K S}(r)=\varepsilon_{i}^{K S} \phi_{i}^{K S}(r)$

$V_{e f f}(r)=V_{e x t}(r)+V_{e e}(r)+V_{x c}[\rho(r)]$

where, $V_{\text {ee }}(r)$ describes the electron-electron interaction (the classical Coulomb interaction) that is defined by:

$V_{e e}(r)=\int d^{3} r^{\prime} \frac{e^{2} \rho\left(r^{\prime}\right)}{4 \pi \in_{0}\left|r-r^{\prime}\right|}$

and $V_{x c}[\rho(r)]$ is the functional derivative of the exchange correlation energy with respect to the electron density function:

$V_{e e}[\rho(r)]=\frac{\delta E_{x c}[\rho(r)]}{\delta \rho(r)}$

The total energy of the atomic system can be arranged as:

$E[\rho]=T_{k}[\rho]+V_{e x t}[\rho]+V_{e e}[\rho]+E_{x c}[\rho]$

where, $T_{k}[\rho]$ represents the kinetic energy of noninteracting electrons. The exchange-correlation functional in Equation (54) can be written as: 


$$
E_{x c}[\rho]=\left(T[\rho]-T_{k}[\rho]\right)+\left(V_{e l}[\rho]-V_{e e}[\rho]\right)
$$

where, $V_{e l}[\rho]$ is non-local electron-electron interaction beyond the classical one in Equation (53). $E_{x c}[\rho]$ is simply the sum of the error made in using a noninteracting kinetic energy and the error made in treating the electron-electron interaction classically. The Kohn-Sham equations in Equation (51) have the same structure as the Hartree-Fock equations with the nonlocal exchange potential replaced by the local exchange-correlation potential $V_{x c}[\rho(r)]$. The computational cost of solving the Kohn-Sham equations scales formally as $\mathrm{N}^{3}$ (due to the need to maintain the orthogonality of $\mathrm{N}$ orbitals), but in current practice it drops to $\mathrm{N}$ through the exploitation of the locality of the orbitals. Actually, the utility of the theory rests on the approximation made for $E_{x c}[\rho]$.

Therefore, the correct description of the exchangecorrelation functional takes a crucial role in DFT. The localdensity approximation has already achieved satisfactory results for systems with a slowly varying electron density function, such as metals (Kohanoff, 2006). However, it has a tendency, termed over-binding, to overestimate binding energies and thus, for instance, predicts too strong hydrogen bonds with too short bond lengths. The generalized gradient approximation is a systematic expansion, which gives good results in most cases and corrects the issue of over-binding (Kohanoff, 2006). Recently, hybrid functionals (Alkauskas et al., 2008) have emerged, which achieve an improved accuracy, especially for semiconductors with a bandgap (Broqvist et al., 2008).

Defect levels for charge capture or emission are calculated by means of the formation energies $E_{f}^{q}\left[X^{q}\right]$, (de Walle and Neugebauer, 2004) with are defined for a certain charge state $\mathrm{q}$ and a certain atomic configuration $X^{q^{\prime}}$ of the defect as:

$$
\begin{aligned}
& E_{f}^{q}\left[X^{q^{\prime}}\right]=E_{\text {tot }}\left[X^{q^{\prime}}\right]-E_{\text {tot }}[\text { bulk }] \\
& -\sum_{j} n_{j} \xi+q\left(\mu+\varepsilon_{v}+\Delta V\right)+E_{c o r r}
\end{aligned}
$$

Here, $E_{t o t}[b u l k]$ stands for the total energy of a supercell containing pure bulk material while $E_{t o t}\left[X^{q}\right]$ represents the super-cell containing a defect. The third term corrects for the different numbers of atoms in both super-cells. The integer $n_{j}$ stands for the number of added $\left(n_{j}>0\right)$ or removed $\left(n_{j}<0\right)$ atoms which are required to create the defect from a perfect bulk structure and $\xi_{j}$ denotes the corresponding energy in an atomic reservoir, which must be specified for each individual case. The fourth term accounts for the charge state $q$ of the defect, in which $\mu$ is defined as the electron chemical potential referenced with respect to the valence band edge $\varepsilon_{v}$ in a bulk-like region and $\Delta V$ corrects the shift in the reference level between charged and uncharged super-cells and is obtained from the difference in the electrostatic potential far distant from the defect for these two super-cells. Due to the periodic boundary conditions, charge neutrality must be maintained within a super-cell. Therefore, a homogeneous compensating background charge must be introduced in calculations of charged defects. This artificial Coulomb interaction is corrected by the last term $E_{\text {corr }}$.

\section{Defect-Assisted Resonant Tunneling}

At low temperatures, the defect-assisted tunneling through thermal emission can be neglected (Huang et al., 1999). Therefore, the whole elastic tunneling process can be divided into two subsequent ones, i.e., tunnel capture and tunnel emission, as shown in Fig. 4. Although the in-plane momentum of electrons is not conserved during the tunneling process, the kinetic energy of electrons is conserved. For a neutral point defect, let us assume that it sits at an arbitrary position $\mathrm{z}=\mathrm{z}_{0}$ inside a barrier layer $\left(0 \leq \mathrm{z}_{0} \leq \mathrm{L}_{\mathrm{B}}\right)$ between the Left (L) and Right (R) electrodes with energy levels $0<E_{d}\left(z_{0}\right)$ $<\Delta E_{c}$, where $\Delta \mathrm{E}_{\mathrm{c}}$ is the conduction band offset for the middle barrier layer. A bias field $\varepsilon_{\mathrm{b}}$ is applied across the layer, leading to a voltage drop $V_{b}=\varepsilon_{b} L_{B}$.

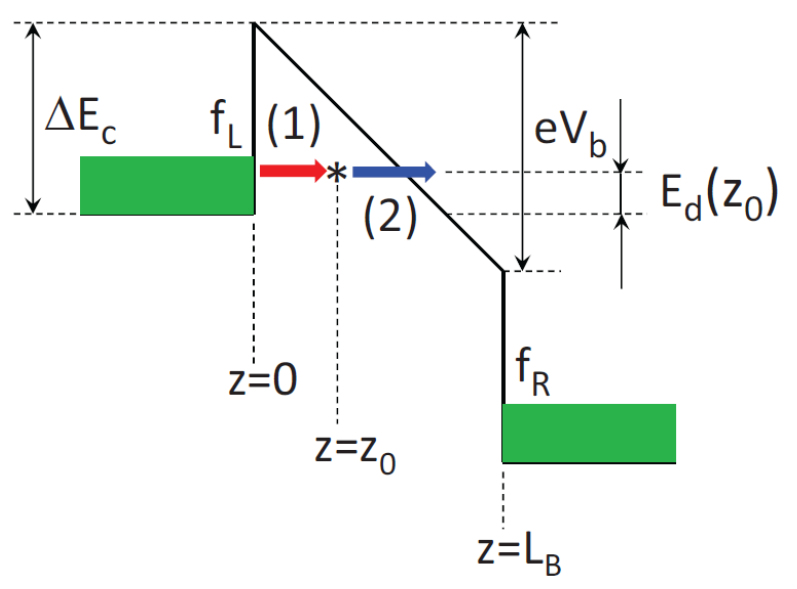

Fig. 4. Schematic of defect-assisted resonant tunneling, where a point defect with energy $\mathrm{E}=\mathrm{E}_{\mathrm{d}}\left(\mathrm{z}_{0}\right)$ at $\mathrm{z}=\mathrm{z}_{0}$ inside the barrier layer with conduction-band offset $\Delta \mathrm{E}_{\mathrm{c}}$ and barrier thickness $L_{B}$. The electron from the left electrode with Fermi distribution $\mathrm{f}_{\mathrm{L}}$ is first captured from left (process-1 in red) by the point defect through tunneling and then emitted to a continuum state above the energy barrier on the right (process- 2 in blue) through tunneling in the presence of a voltage drop $\mathrm{V}_{\mathrm{b}}$ across the barrier layer 
By assuming a large voltage drop, we need to consider only the forward current from left to right but not the backward current from right to left. In this picture, the left-going (capture) tunneling current density $J_{L}\left(V_{b}, T\right)$ can be formally written as (Huang et al., 2008):

$$
\begin{aligned}
& J_{L}\left(V_{b}, T\right)=2 e \int_{0}^{L_{B}} d z_{0} \rho_{d}\left(z_{0}\right) \\
& \sum_{k} \frac{2 \pi}{h}\left|\left\langle\Psi_{k}\left|U_{d}\right| \psi_{d}\right\rangle\right|^{2} \frac{\Gamma_{d} / \pi}{\left[E_{k}-E_{d}\left(z_{0}\right)\right]^{2}+\Gamma_{d}^{2}} F_{L}\left(E_{k}\right)
\end{aligned}
$$

where, $\rho_{d}\left(z_{0}\right)$ represents the distribution of the pointdefect concentration, $U_{d}(r)$ is the Coulomb potential associated with the point defect, $\psi_{d}(r)$ is its wave function and $\Gamma_{d}$ is the broadening in the density of states for point defects.

In Equation (58), the occupation factor $F_{L}\left(E_{k}\right)$ is defined as:

$$
\begin{aligned}
& F_{L}\left(E_{k}\right)=f_{L}^{(0)}\left(E_{k}\right)\left\{1-g\left[E_{d}\left(z_{0}\right)\right]\right\} \\
& Z_{e}-\left[1-f_{L}^{(0)}\left(E_{k}\right)\right] g\left[E_{d}\left(z_{0}\right)\right] Z_{f}
\end{aligned}
$$

where, $E_{k}=h^{2} k^{2} / 2 m^{*}$ is the electron kinetic energy with effective mass $\mathrm{m} *$ in the left electrode, $Z_{e}$ and $Z_{f}$ represent the structural degeneracy factors of the point defect when empty or filled, $g\left[E_{d}\left(z_{0}\right)\right]$ is the defect occupancy function

and $f_{L}^{(0)}\left(E_{k}\right)=\left\{1+\exp \left[\left(E_{k}-\mu_{0}\right) / k_{B} T\right]\right\}^{-1} \quad$ is the Fermi distribution function in the left electrode with chemical potential $\mu_{0}$. In addition, by employing the WKB approximation (Gill, 1982) for the electron wave function $\Psi_{k}(r)$, the interaction matrix $\left\langle\Psi_{k}\left|U_{d}\right| \psi_{d}\right\rangle$ is calculated as (Stievenard et al., 1992):

$$
\left\langle\Psi_{k}\left|U_{d}\right| \psi_{d}\right\rangle=\frac{A_{k}}{\sqrt{K\left(z_{0}\right)}} \exp \left[-\int_{0}^{z_{0}} d z^{\prime} K\left(z^{\prime}\right)\right] u_{1}\left[K\left(z_{0}\right), k_{\|}\right]
$$

where, $A_{k}$ is an unknown coefficient to be determined by the continuity of the wave function at the boundaries, $S$ is the cross-sectional area:

$$
\begin{aligned}
& K(z)=\frac{\sqrt{2 m_{2}^{*}}}{\hbar}\left[\Delta E_{c}-\frac{e V_{b^{z}}}{L B}\right] \\
& u_{1}\left[K\left(z_{0}\right), k_{\|}\right] \\
& =\int d^{3} r \psi_{d}(r) U_{d}(r)\left[\frac{K\left(z_{0}\right)}{K(z)}\right]^{1 / 2} \frac{e^{-i k} \| \cdot r_{\|}}{\sqrt{S}} \exp \left[-\int_{z_{0}}^{z} d z^{\prime} K\left(z^{\prime}\right)\right]
\end{aligned}
$$

In a similar way, we can also calculate the rightgoing (escape) tunneling current density $J_{R}\left(V_{b}, T\right)$. In steady state, we have $J_{L}\left(V_{b}, T\right)=-J_{R}\left(V_{b}, T\right) \equiv J\left(V_{b}, T\right)$. This allows us to eliminate the unknown defect occupancy function $g\left[E_{d}\left(z_{0}\right)\right]$ and eventually obtain (Stievenard et al., 1992):

$$
\begin{aligned}
& J\left(V_{b}, T\right)=2 e Z_{e} Z_{f}\left(f_{L}^{(0)}-f_{R}^{(0)}\right) \\
& \int_{0}^{L_{B}} d z_{0} \rho d\left(z_{0}\right)\left[\frac{\Theta_{R}}{P_{c}\left(z_{0}\right)}+\frac{\Theta_{L}}{P_{e m}\left(z_{0}\right)}\right]
\end{aligned}
$$

where, $\quad f_{R}^{(0)}\left(E_{k}\right)=\left\{1+\exp \left[\left(E_{k}-\mu_{0}+e V_{b}\right) / k_{B} T\right]\right\}^{-1} \quad$ and $\Theta_{L, R}=f_{L, R}^{(0)} Z_{e}+\left(1-f_{L, R}^{(0)}\right) Z_{f}$. In addition, the tunnel-capture rate (probability) $P_{c}\left(z_{0}\right)$ of an electron by a point defect in Equation (63) is defined as:

$$
P_{c}\left(z_{0}\right)=\frac{2 \pi}{\hbar} \sum_{k}\left|\left\langle\Psi_{k}\left|U_{d}\right| \psi_{d}\right\rangle\right|^{2} \frac{\Gamma_{d} / \pi}{\left[E_{k}-E_{d}\left(z_{0}\right)\right]^{2}+\Gamma_{d}^{2}}
$$

and the tunnel-emission rate (probability) $P_{e m}\left(z_{0}\right)$ of an electron captured by a strongly-localized point defect is given by:

$$
P_{e m}\left(z_{0}\right)=\frac{e \varepsilon_{b}}{4 \sqrt{2 m_{2}^{*} E_{d}\left(z_{0}\right)}} \exp \left(-\frac{4 \sqrt{2 m_{2}^{*} E_{d}^{3}\left(z_{0}\right)}}{3 e \hbar \varepsilon_{b}}\right)
$$

For photo-detectors, the defect-assisted resonant tunneling greatly increases the dark current in the absence of incident light, which generates excess noise and reduces the detectivity of the photo-detector (Huang et al., 2000).

\section{Reduced Carrier Mobility}

When point defects are charged with a charge number $|Z *| \geq 1$, they can scatter conduction electrons through their Coulomb potential $\sum_{i=1}^{N} \int d^{3} r^{\prime} U_{c}\left(r-r^{\prime}\right)\left|\psi_{d}\left(r^{\prime}-r_{i}\right)\right|^{2}$, as shown in Fig. 5, where $r_{i}$ for $\mathrm{i}=1,2, \ldots, \mathrm{N}$ represent the positions of $\mathrm{N}$ point defects inside the quantum well (formed in semiconductors by having a layer of narrow bandgap material sandwiched between two layers of a material with a wider bandgap to create discrete energy levels) and $\psi_{d}(r) \equiv \psi_{d}\left(r_{\Lambda}\right) \gamma_{d}(z)$ is the wave function of the point defect in layered semiconductors. Let us consider electrons confined in one of the quantum wells with width $\mathrm{L}_{\mathrm{W}}$ and barrier height $\Delta \mathrm{E}_{\mathrm{c}}$. For simplicity, we assume that only the ground state of electrons is occupied at low temperatures with the wave function $\psi_{1 k_{\|}}(r)=\frac{e^{i k_{\|} \cdot r_{\|}}}{\sqrt{S}} \phi_{1}(z)$ and subband energy $E_{1}\left(k_{\|}\right)=\varepsilon_{1}+h^{2} k_{\|}^{2} / 2 m^{*} \quad$ with quantum-well crosssectional area $\mathrm{S}$, subband edge $\varepsilon_{1}$ and electron effective mass $\mathrm{m}^{*}$. 


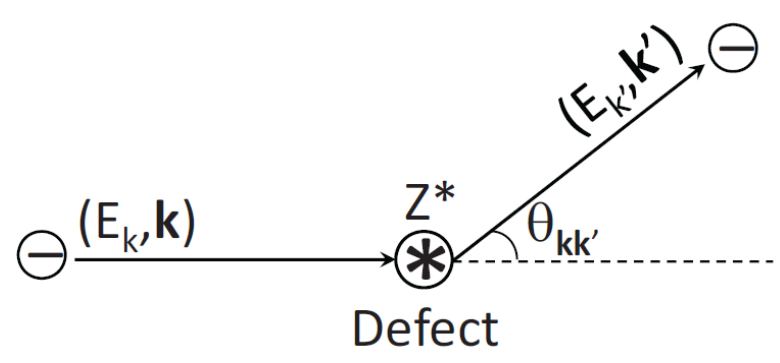

Fig. 5. Schematic of scattering of an electron by a point defect, where the defect is charged and has an effective charge number $Z^{*}$. The incident electron with wave vector $\mathrm{k}$ and kinetic energy $E_{k}$ is scattered into a different direction with wave vector $\mathrm{k}^{\prime}$ and kinetic energy $\mathrm{E}_{\mathrm{k}^{\prime}}$. The scattering angle between $\mathrm{k}$ and $\mathrm{k}^{\prime}$ is denoted by $\theta_{\mathrm{kk}^{\prime}}$ and the elastic scattering process requires $\mathrm{E}_{\mathrm{k}}=\mathrm{E}_{\mathrm{k}^{\prime}}$

In this case, the interaction matrix $\left\langle\Psi_{1 k_{\| 1}}\left|U_{c}\right| \Psi_{1 k^{\prime} \|}\right\rangle$ is calculated as (Huang et al., 2005a):

$$
\begin{aligned}
& \left\langle\Psi_{1 k_{\|}}\left|U_{c}\right| \Psi_{1 k_{\|}^{\prime}}\right\rangle \\
& =\int d z\left|\phi_{1}(z)\right|^{2} \sum_{i=1}^{N} \sum_{q_{\|}} U_{c}\left(q_{\|}, z-z_{i}\right) F_{d}\left(q_{\|}\right) \delta_{q \|}, k_{\|}-k_{\|}^{\prime} e^{-i q\|\cdot \cdot r\|}
\end{aligned}
$$

where, the two-dimensional Fourier transform of $U_{c}\left(r-r_{i}\right)$ is denoted as $U c\left(q_{1}, z-z_{i}\right)$ and give by:

$$
\begin{aligned}
& U_{c}\left(q_{\|}, z-z_{i}\right)=\left[\frac{ \pm Z^{*} e^{2}}{2 \in_{0} \in_{r}\left(q_{\|}+q_{s}\right)}\right] \\
& \int d z^{\prime} e^{-q \| z^{2}-z_{i}-z^{\prime}}\left|\gamma d\left(z^{\prime}\right)\right|^{2} \\
& F_{d}\left(q_{\|}\right)=\int d^{2} r_{\|} e^{-i q\|\cdot r\|}\left|\psi_{d}\left(r_{\|}\right)\right|^{2}
\end{aligned}
$$

and $\epsilon_{r}$ is the dielectric constant of the quantum-well host material. Moreover, $q_{s}$ in Equation (67) is the inverse Thomas-Fermi screening length for quantumwell electrons, given by (Huang and Manasreh, 1996):

$$
q_{s}=\frac{e^{2}}{8 \pi \in_{0} \in_{r} k_{B} T} \int_{0}^{\infty} d k_{\|} k_{\|} \cosh ^{-2}\left[\frac{E_{1}\left(k_{\|}\right)-\mu_{0}}{2 k_{B} T}\right]
$$

where, $\mathrm{T}$ is the electron temperature and $\mu_{0}$ is the chemical potential of electrons in the quantum well.

Since the positions of point defects are random, by introducing a continuous linear density distribution $\rho_{l d}\left(z_{0}\right)=S_{\rho d}\left(z_{0}\right)$ for point defects, the interaction matrix from Equation (66) becomes:

$$
\begin{aligned}
& \left|\left\langle\Psi_{1 k \|}\left|U_{c}\right| \Psi 1 k_{\|}^{\prime}\right\rangle\right|^{2}=\left[\frac{Z^{*} e^{2}}{2 \epsilon_{0} \in_{r}\left(\left|k_{\|}-k_{\|}^{\prime}\right|+q_{s}\right)}\right]^{2}\left|F_{d}\left(\left|k_{\|}-k_{\|}^{\prime}\right|\right)\right|^{2} \\
& \times \int_{-L w / 2}^{L_{W}} d z_{0} \rho_{1 d}\left(z_{0}\right)\left|\left(\int_{-\infty}^{\infty} d z\left|\phi_{1}(z)\right|^{2} \int d z^{\prime} e^{-\left|k_{\|}\left\|k_{\|}^{\prime}\right\| z^{-z_{0}-z^{\prime}}\right|}\left|\gamma d\left(z^{\prime}\right)\right|^{2}\right)\right|^{2}
\end{aligned}
$$

where, $L_{W}$ is the width of the quantum well. Once the scattering matrix elements in Equation (70) are computed, by using Fermi's golden rule, the momentumrelaxation time $\tau_{0}$ can be obtained from:

$$
\begin{aligned}
& \frac{1}{\tau_{0}}=\frac{1}{2 \pi \hbar} \int d^{2} k_{\|} \int d^{2} k_{\|}^{\prime}\left|\left\langle\Psi_{1 k_{\|}}\left|U_{c}\right| \Psi_{1 k_{\|}^{\prime}}\right\rangle\right|^{2} \\
& \delta\left[E_{1}\left(k_{\|}\right)-E_{1}\left(k_{\|}^{\prime}\right)\right]\left(1-\cos \theta_{k_{\|} k_{\|}^{\prime}}\right)
\end{aligned}
$$

where, $\theta_{\left.k_{\|}\right|_{\|} ^{\prime}}$ represents the angle between the two inplane scattering wave vectors $k_{\|}$and $k_{\|}^{\prime}$. By using the momentum-relaxation $\tau_{0}$ in Equation (71), the mobility $\mu_{e}$ of electrons can be simply expressed as $\mu_{e}=\frac{e \tau_{0}}{m^{*}}$. The reduced mobility of conduction carriers by radiation-induced point defects will directly affect the speed of high-mobility field-effect transistors in an integrated circuit (Ando et al., 1982).

\section{Non-Radiative Recombination with Defects}

After the electrons are photo-excited from valence band to conduction band in a semiconductor, some of these photo-electrons will be quickly captured by point defects through an inelastic scattering process, (Huang et al., 2008) as shown in Fig. 6. By including the multi-phonon emission at room temperature (Huang et al., 2005b; Ridley, 1978), in this case the capture rate is calculated as (Jim'enez-Molinos et al., 2001; Garetto et al., 2012):

$$
\begin{aligned}
& W_{c}^{e, h}\left(k, z_{0}\right)=\frac{2 \pi}{\hbar}\left|\left\langle\psi_{d}\left|U_{\{e, h\} p}\right| \Psi_{k}^{e, h}\right\rangle\right|^{2} \beta_{H R}\left[\frac{1-\Delta E_{e, h}\left(z_{0}\right)}{h \Omega_{0} \beta_{H R}}\right]^{2} \\
& \times E X P\left[-\left[2 N_{p h}\left(\Omega_{0}\right)+1\right] \beta_{H R}+\frac{\Delta E_{e, h}\left(z_{0}\right)}{2 k_{B} T}\right] \\
& \sum_{m=1}^{\infty} \mathrm{I}_{\mathrm{m}}(\xi) \frac{\Gamma_{d} / \pi}{\left[m \hbar \Omega_{0}-\Delta E_{e, h}\left(z_{0}\right)+\Gamma_{d}^{2}\right]}
\end{aligned}
$$

where, $U_{\{e, \quad h\} p}(r)$ represent the potentials for the electron-phonon and hole-phonon coupling, $\psi_{\mathrm{d}}(\mathrm{r})$ is the wave function of the point defect, $\Gamma_{d}$ is the level broadening of the defect state, $\Psi_{k}^{e, h}(r)$ are the wave functions of electrons (e) and holes (h) in a bulk, $\beta_{\mathrm{HR}}$ is the Huang-Ryhs factor, $\Delta E_{e}\left(z_{0}\right)=E_{G}+E_{k}^{e}-E_{d}\left(z_{0}\right), \Delta E_{h}\left(z_{0}\right)=E_{d}\left(z_{0}\right)+E_{k}^{h}, E_{k}^{e, h} \quad$ are the kinetic energies of electrons and holes, $E_{G}$ is the bandgap energy of the semiconductor, $\mathrm{h} \Omega_{0}$ is the opticalphonon $N_{p h}\left(\Omega_{0}\right)=\left[\exp \left(h \Omega_{0} / k_{B} T\right)-1\right]^{1}$ is the distribution function of thermal-equilibrium phonons, $\mathrm{T}$ is the temperature and $I_{m}(\xi)$ is the modified Bessel function of order m with $\xi=2 \beta_{H R} \sqrt{N_{p h}\left(\Omega_{0}\right)\left[N_{p h}\left(\Omega_{0}\right)+1\right]}$. 


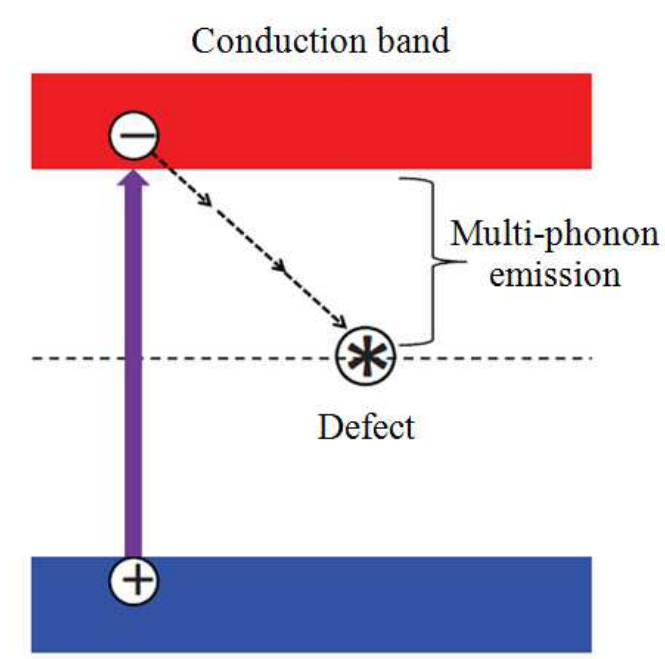

Valence band

Fig. 6. Schematic of non-radiative recombination of photoexcited electrons from valence band to conduction band with point defects, where multiple phonons are emitted while the photo-excited electrons recombine with localized defect states within the bandgap

The electron-phonon coupling matrix element $\left|\left\langle\psi_{d}\left|U_{\{e, h\} p}\right| \Psi_{k}^{e, h}\right\rangle\right|^{2}$ in Equation (72) can be evaluated by (Huang and Alsing, 2008):

$$
\left|\left\langle\psi_{d}\left|U_{\{e, h\} p}\right| \Psi_{k}^{e, h}\right\rangle\right|^{2}=\frac{1}{(2 \pi)^{3}} \int d^{3} q\left|B_{d}(q-k)\right|^{2}\left|U_{\{e, h\} p}(q)^{2}\right|
$$

where,

$$
\begin{aligned}
& B_{d}(q-k)=\int d^{3} r \psi_{d}(r) e^{i(q-k) \cdot r} \\
& \left|U_{\{e, h\} p}(q)^{2}\right|=\frac{\hbar \Omega_{0}}{2}\left(\frac{1}{\epsilon_{\infty}}-\frac{1}{\epsilon_{s}}\right) \frac{e^{2}}{\epsilon_{0}\left(q^{2}+Q_{e, h}^{2}\right) V}
\end{aligned}
$$

$\mathrm{V}$ is the system volume, $\epsilon_{\infty}$ and $\epsilon_{s}$ are the highfrequency and static dielectric constants of the host semiconductor and the inverse Thomas-Fermi screening length $Q_{e, h}$ for bulk electrons and holes is given by:

$$
\begin{aligned}
& Q_{e, h}^{2}=\frac{e^{2}}{\pi^{2} \in_{0} \in_{r} k_{B} T} \int_{0}^{\infty} d k k^{2} f_{0}\left(E_{k}^{e, h}-\mu_{e, h}\right) \\
& {\left[1-f_{0}\left(E_{k}^{e, h}-\mu_{e, h}\right)\right]}
\end{aligned}
$$

Here, $f_{0}\left(E_{k}^{e, h}-\mu_{e, h}\right)=\left\{1+\exp \left[\left(E_{k}^{e, h}-\mu_{e, h}\right) / k_{B} T\right]\right\}^{-1}$ is the Fermi distribution function for thermal-equilibrium conduction electrons and holes with chemical potentials $\mu_{\mathrm{e}, \mathrm{h}}$.
Finally, based on the given expression for $\left|\left\langle\psi_{d}\left|U_{\{e, h\} p}\right| \Psi_{k}^{e, h}\right\rangle\right|^{2}$ in Equations (73)-(75), the rate for the non-radiative recombination $\frac{1}{\tau_{n r}^{e, h}}$ can be explicitly calculated from:

$\frac{1}{\tau_{n r}^{e, h}}=\int d z_{0} \rho 1 d\left(z_{0}\right)\left[\frac{1}{\tau_{d}^{e, h}\left(z_{0}\right)}\right]$

$\left[\begin{array}{c}1 / \tau_{d}^{e}\left(z_{0}\right) \\ 1 / \tau_{d}^{h}\left(z_{0}\right)\end{array}\right]=\left[\begin{array}{c}\left\{1-g\left[E_{d}\left(z_{0}\right)\right]\right\} Z_{e} W_{e}\left(z_{0}\right) \\ g\left[E_{d}\left(z_{0}\right)\right] Z_{f} W_{h}\left(z_{0}\right)\end{array}\right]$

$W_{e, h}\left(z_{0}\right)=\int \frac{d^{3} k}{(2 \pi)^{3}} W_{c}^{e, h}\left(k, z_{0}\right) f_{0}\left(E_{k}^{e, h}-\mu_{e, h}\right)$

$W_{e, h}\left(z_{0}\right)=\Re_{\mathrm{e}, h}\left(z_{0}\right) \int \frac{d^{3} q}{(2 \pi)^{3}}\left[\frac{e^{2}}{\epsilon_{0}\left(q^{2}+Q_{e, h}^{2}\right)}\right]\left|B_{d}(q-k)\right|^{2}$

$\times \sum_{m=1}^{\infty} \mathrm{I}_{\mathrm{m}}(\xi) \frac{\Gamma_{d} / \pi}{\left[m-\Delta E_{e, h}\left(z_{0}\right) / \hbar \Omega_{0}\right]^{2}+\Gamma_{d}^{2}}$

where, $\rho_{l d}\left(z_{0}\right)=S \rho_{d}\left(z_{0}\right)$ is the linear density distribution of point defects in a layer with the crosssectional area $\mathrm{S}, \mathrm{g}\left[\mathrm{E}_{\mathrm{d}}\left(\mathrm{z}_{0}\right)\right]$ in Equation (78) is the defect occupancy function and $Z_{e}$ and $Z_{f}$ represent the structural degeneracy factors of $\frac{1}{\tau_{d}^{e}\left(z_{0}\right)}=\frac{1}{\tau_{d}^{h}\left(z_{0}\right)}=\frac{1}{\tau_{d}\left(z_{0}\right)}$ which allows us to eliminate the unknown $g\left[E_{d}\left(z_{0}\right)\right]$ introduced in Equation (78), similar to what we have done in deriving Equation (63). This leads to:

$$
\begin{aligned}
& \frac{1}{\tau_{n r}}=\frac{1}{\tau_{n r}^{e}}=\frac{1}{\tau_{n r}^{h}}=Z_{e} Z_{f} \int d z_{0} \rho 1 d\left(z_{0}\right) \\
& {\left[\frac{W_{e}\left(z_{0}\right) W_{h}\left(z_{0}\right)}{Z_{e} W_{e}\left(z_{0}\right)+Z_{f} W_{h}\left(z_{0}\right)}\right]}
\end{aligned}
$$

The change in the non-radiative time by point defects in the system will reduce the quantum efficiency of photo-excited electrons in both light-emitting diodes and photo-detectors (Huang and Lyo, 1999).

\section{Inelastic Light Scattering by Charged Defects}

Let us choose the $\mathrm{z}$ direction perpendicular to the layered material. Light is incident on the layers in the xyplane and scattered by charged point defects within the layers. We consider incident light with photon energy $h \omega_{i}$ and wave vector $\mathrm{k}_{\mathrm{i}}$ scattered inelastically by bound electrons within point defects at $r_{j}=\left(r_{j \|}, z_{j}\right)$ for $\mathrm{j}=1,2, \ldots$. If the scattered-light photon energy and wave vector are 
denoted by $h \omega_{\mathrm{f}}$ and $\mathrm{k}_{\mathrm{f}}$, respectively, the excitation energy and momentum transfer to charged point defects are given by $h w=h w_{f}-h w_{i}$ and $h_{q}=h k_{f}-h k_{i}$. We further assume that the ground and excited state (real) wave functions of defects are expressed as $\psi_{d}^{(0)}\left(r-r_{j}\right)=\psi_{d}^{(0)}\left(r_{\|}-r_{j \|}\right) \gamma_{d}^{(0)}\left(z-z_{j}\right) \quad$ and $\psi_{d}^{(n)}\left(r-r_{j}\right)=\psi_{d}^{(n)}\left(r_{\|}-r_{j \|}\right) \gamma_{d}^{(n)}\left(z-z_{j}\right)$, where $\mathrm{n}=1,2 \ldots$ represent different excited states of a charged point defect. The energy levels for the ground and excited states of charged defects are separately represented by $E_{d}^{(0)}\left(z_{j}\right)$ and $E_{d}^{(n)}\left(z_{j}\right)$.

In a standard way, the differential scattering cross section $\frac{d^{2} \sigma(q, w)}{d w d \Omega_{q}}$ for inelastic light scattering can be shown to be (Zhang et al., 1991):

$$
\begin{aligned}
& \frac{d^{2} \omega(q, w)}{d w d \Omega_{q}}=\left(\frac{e^{2}}{4 \pi \epsilon_{0} m^{*} c^{2}}\right)^{2} \hbar\left|e_{i} \cdot e_{f}\right|^{2}\left(\frac{\omega_{i}}{\omega_{f}}\right) \frac{N_{p h}(\omega)+1}{\pi} \\
& \times \int d z_{0} \rho 1 d\left(z_{0}\right) \operatorname{Im}\left[\sum_{n, n^{\prime}} A_{n n^{\prime}}\left(q_{\|}, q_{z} \mid z_{0}\right) Q_{n n^{\prime}}\left(q, \omega \mid z_{0}\right)\right]
\end{aligned}
$$

where, $q=\left(q_{\|}, q_{z}\right), \mathrm{e}_{\mathrm{i}}$ and $\mathrm{e}_{\mathrm{f}}$ are the unit polarization vectors for the incident and scattered light, $\mathrm{N}_{\mathrm{ph}}(\mathrm{w})=$ $\left[\exp \left(\mathrm{hw} / \mathrm{k}_{\mathrm{B}} \mathrm{T}\right)-1\right]^{-1}$ is the photon distribution function, $\mathrm{T}$ is the temperature, $\Omega_{q}$ represents the solid angle in threedimensional q-space and $\rho_{1 \mathrm{~d}}\left(\mathrm{z}_{0}\right)$ is the linear density of charged point defects.

In addition, the form factor $A_{n n^{\prime}}\left(q_{\|}, q_{z} \mid z_{0}\right)$ introduced in Equation (83) is calculated as:

$$
\begin{aligned}
& A_{n n^{\prime}}\left(q_{\|}, q_{z} \mid z_{0}\right)=e^{-2 i q_{z} z 0} \\
& \int d^{2} r_{\|} \int d^{2} r_{\|}^{\prime} \psi_{d}^{(n)}\left(r_{\|}\right) e^{i q_{\|} \cdot\left(r_{\|}-r_{\|}^{\prime}\right)} \psi_{d}^{\left(n^{\prime}\right)}\left(r_{\|}^{\prime}\right) \psi_{d}^{(0)}\left(r_{\|}^{\prime}\right) \\
& \times \int d z \int d z^{\prime} \gamma_{d}^{(n)}(z) \gamma_{d}^{(0)}(z) e^{-i q_{z}\left(z+z^{\prime}\right)} \gamma_{d}^{\left(n^{\prime}\right)}\left(z^{\prime}\right) \gamma_{d}^{(0)}\left(z^{\prime}\right)
\end{aligned}
$$

The interacting density-density correlation function $Q_{n n^{\prime}}\left(q, w \mid z_{0}\right)$ employed in Equation (83) is:

$$
Q_{n n^{\prime}}\left(q, \omega \mid z_{0}\right)=\sum_{m} \epsilon_{n m}^{-1}(q, \omega) \Pi_{\mathrm{m} n^{\prime}}^{(0)},\left(\omega \mid z_{0}\right)
$$

where, $\epsilon_{n m}^{-1}(q, w)$ represents the matrix element of the inverse dielectric function of the host material containing defects. In addition, $\Pi_{\mathrm{m} n^{\prime}}^{(0)}\left(\omega \mid z_{0}\right)$ in Equation (85) is the non-interacting density-density correlation function, given by:

$$
\Pi_{n n^{\prime}}^{(0)}\left(\omega \mid z_{0}\right)=\delta_{n^{\prime}, 0} \frac{2 n_{d}^{(0)}\left(T \mid z_{0}\right)\left[E_{d}^{(n)}\left(z_{0}\right)-E_{d}^{(0)}\left(z_{0}\right)\right]}{\left[E_{d}^{(n)}\left(z_{0}\right)-E_{d}^{(0)}\left(z_{0}\right)\right]^{2}-\hbar^{2} \omega\left(\omega+i \Gamma_{d}\right)}
$$

where, we assume that only the ground state of charged point defects is occupied with the thermal occupation factor $n_{d}^{(0)}\left(T \mid z_{0}\right)$.

If there exist conduction electrons, in addition to bound electrons in charged point defects, with concentration $n_{0}$, effective mass $\mathrm{m} *$ and homogeneous broadening $\gamma_{\mathrm{e}}$ in the host material containing generated point defects, the matrix elements of the dielectric function are found to be (Huang et al., 2006):

$$
\epsilon_{n m}(q, w)=\delta_{m, n}\left[1-\frac{\Omega_{p}^{2}}{\omega\left(\omega+i \gamma_{e}\right)-3 v_{F}^{2} q^{2} / 5}\right]
$$

where, $v_{F}=\mid\left(h / m^{*}\right)\left(3 \pi^{2} n_{0}\right)^{l / 3}$ is the Fermi velocity of conduction electrons at zero temperature, $\Omega_{p}=$ $\left(n_{0} e^{2} / \epsilon_{0} \epsilon_{b}\right)^{1 / 2}$ is the plasma frequency and $\epsilon_{\mathrm{b}}$ is the dielectric constant of the host material.

Furthermore, if the host material is a doped polar semiconductor, its optical phonon modes can couple to conduction electrons. In this case, the matrix elements of the dielectric function in Equation (87) are modified to (Huang and Zhou, 1988):

$$
\epsilon_{n m}(q, \omega) \rightarrow \delta_{m, n}\left\{\begin{array}{l}
1-\left[\frac{\omega\left(\omega+i \gamma_{p}\right)-\Omega_{T O}^{2}}{\omega\left(\omega+i \gamma_{p}\right)-\Omega_{L O}^{2}}\right] \\
\frac{\Omega_{p}^{2}}{\omega\left(\omega+i \gamma_{e}\right)-3 v_{F}^{2} q^{2} / 5}
\end{array}\right\}
$$

where, the static dielectric constant $\epsilon_{b}$ in the expression for $\Omega_{p}$ should be replaced with the optical-frequency one $\epsilon_{\infty}, \Omega_{L O}$ and $\Omega_{T O}$ are the frequencies of the longitudinal and transverse optical phonon modes and $\gamma_{p}$ represents the phonon homogeneous broadening.

The inelastic-light scattering technique can be used for identifying the charged point-defect species and their electronic properties, (Platzman and Wolff, 1973) such as level separation between ground and excited states, broadening in the defect density of states and optical polarization properties of point defects. If the incident coherent light is provided by a pulsed laser, the ultra-fast dynamics of charged point defects can be directly measured and analyzed (Tsen et al., 2001).

\section{Application of Defect Theory}

Infrared imaging from space typically places the most stringent performance requirements on the hybridized Focal Plane Array (FPA) due to the additional cost, complexity and remoteness the space environment imparts as well as the 3-5 times smaller incident optical flux levels that occur in strategic environments compared to tactical (terrestrial) environments. Space also places the additional, specifically unique, requirement of radiation tolerance or rad-hardness on both elements of the FPA, the Si-CMOS (complementary metal-oxide- 
semiconductor) Read-Out Integrated Circuit (ROIC) and the detector array. FPAs developed for imaging applications in a space environment are meticulously characterized to evaluate their sensitivity, uniformity, operability and radiation hardness. Infrared (IR) hybrid detector arrays operated in the space environment may be subjected to a variety of radiation sources while in orbit. This means IR detectors intended for applications such as space-based surveillance or space-situational awareness must not only have high performance (high quantum efficiency and low dark current density), but also their radiation tolerance, or ability to withstand the effects of the radiation they would expect to encounter in a given orbit, must also be characterized.

Pursuit of these performance metrics are intended to enhance technology development in multispectral sensors, higher pixel sensitivities, larger array formats with higher pixel densities, higher operating temperatures, simplified manufacturing techniques and reduced costs. Specific to space applications, as described above, is radiation hardness or tolerance, which is required to mitigate detrimental effects on IR detectors exposed to energetic charged particles (mostly electrons and protons and some heavy ions) confined by the earth's magnetic field (the van Allen radiation belts), galactic cosmic ray particles (which can be a range of elements or electromagnetic radiation) and particles, protons and heavy ions from solar flares. Detector technologies that operate in the harsh radiation environment of space with better radiation tolerance afford greater flexibility in orbit selection, technical applications and system sustainability and thus, are of more value to the space-based sensing community.

While specific mission requirements for rad-hardness vary, the effects of proton interactions with hybrid detector arrays tend to dominate in space. Thus, a specific detector's degree of radiation tolerance is typically first characterized by measuring its performance degradation as a function of proton irradiation fluence (Hubbs et al., 2007). Subjecting IR detectors to protons will lead to two distinct damage mechanisms, ionization damage and displacement damage, which are expected to account for the performance degradation due to irradiation that will occur in optical sensors in satellites. Ionization damage, or so-called Total Ionizing Dose (TID), effects occur as the incoming particles incident on the FPA give up their kinetic energy to ionizing additional electron-hole pairs within both the detector material and the CMOS silicon of the ROIC. Some of these excess carriers can become trapped in surface states and defect levels of the dielectric materials used for passivation and as gate oxide layers. These surface states and defect levels are to be determined with the theoretical approach presented in this study. The excess trapped charge manifests in flatband voltage shifts of the ROIC's MOSFETs (metaloxide-semiconductor field-effect transistors) and excess leakage currents in the detector pixels, typically surface- currents. Displacement damage results when the incoming particle's energy is lost to elastic or inelastic Coulomb scattering with an atomic nucleus that is sufficient to knock the atom from its lattice site and generate a vacancy-interstitial pair. The defect complexes so formed can act as electrically active sites where electron-hole recombination might occur (Claeys and Simoen, 2013). The additional defect generation thus shortens the detector material's minority carrier recombination lifetime, resulting in increased dark current, decreased responsivity and overall degraded uniformity.

Radiation tolerance characterization typically includes determining the rate of performance degradation via a damage factor analysis (Cowan et al., 2012; Morath et al., 2015). The degradation rates of each measured parameter of the detector's performance [dark current-including the diffusion-limited, Shockley-ReedHall (SRH) generation-recombination and trap-assisted tunneling mechanisms; lateral optical collection length or effective diffusion length-and thus minority-carrier recombination lifetime and mobility; quantum efficiency; etc.] are determined by plotting them as a function of the proton irradiation fluence $\Phi_{P}$ and characterizing the changes. When the change in parameter $\mathrm{X}$ appears roughly linear with $\Phi_{P}$, which may be true on average or for only a certain fluence range, then a damage factor, $\mathrm{K}_{\mathrm{X}}$, can be defined such that $\mathrm{X}=X_{0} \pm K_{X} \Phi_{P}$, where $\mathrm{X}_{0}$ is the un-irradiated value of the performance parameter and the \pm is determined by the expected change, e.g., + for dark current (Fig. 7) and -for quantum efficiency (Fig. 8). Damage factors are assumed to be related only to changes due to the effects of displacement damage, not ionization damage and are dependent on the proton energy E (Claeys and Simoen, 2013).

The radiation-induced defects can manifest in lower quantum efficiency $(\eta)$ and higher dark current density $\left[J_{D}\right.$ which is related to the tunneling current density in Equation (63)], due to the consequent reduction in minority carrier recombination lifetime $\left[\tau_{R}\right.$ which is related to the nonradiative recombination time $\tau_{n r}$ in Equation (77)] and increase in SRH generation-recombination, carrier diffusion and trap-assisted tunneling. Thus, a substantial reduction in overall detector sensitivity, or "detectivity" (D*), is expected. Radiation tolerance can be characterized from performance measurements taken versus fluence by calculating the above-described damage factor $\left(\mathrm{K}_{\mathrm{X}}\right)$ or the rate of degradation for each performance metric $\mathrm{X}$ (e.g., $\mathrm{X}$ $=\eta, 1 / \tau_{R}, J_{D}, D^{*}$, etc). For comparison purposes, it is worth noting that damage factors are specific to the particle type and energy of the incoming radiation. With a known energydependence, $\mathrm{K}_{\mathrm{X}}(\mathrm{E})$, predictions of the expected onorbit degradation $\Delta \mathrm{X}$ ideally become possible, according to:

$\Delta X=\int_{E_{1}}^{E_{2}} d E K_{X}(E) \frac{d \Phi_{p}(E)}{d E}$ 


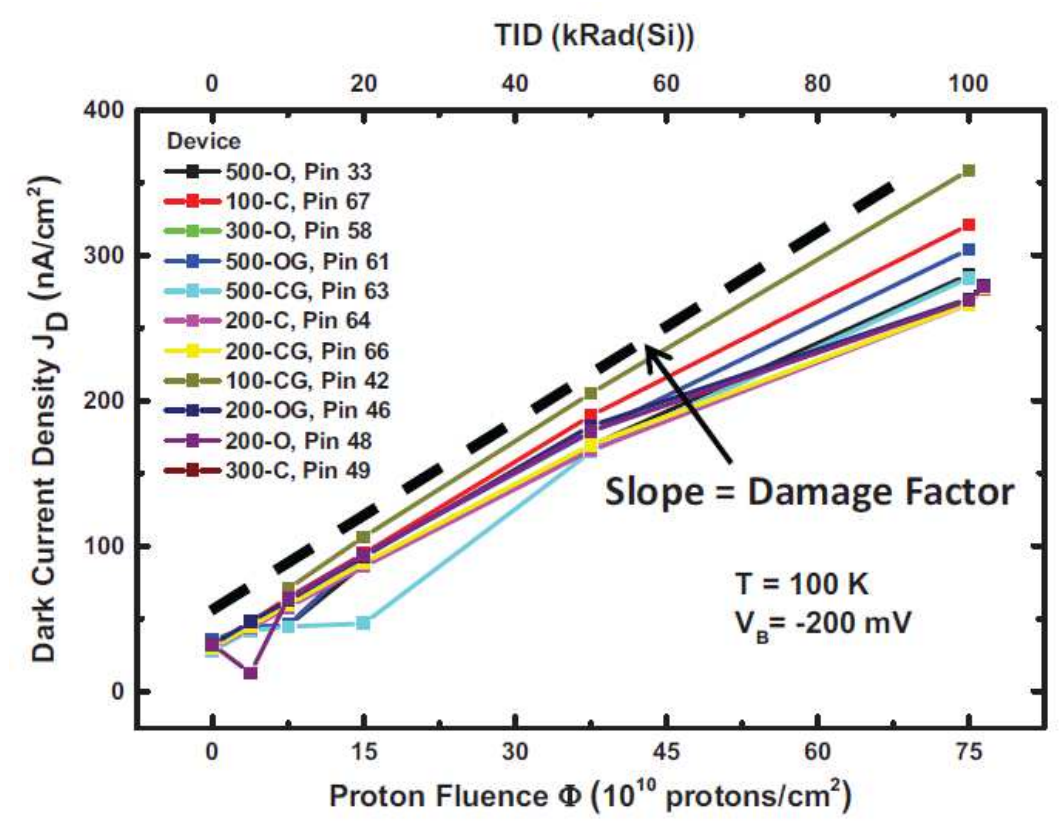

Fig. 7. Dark current (JD) as a function of proton fluence. Dashed line gives a + slope for the trend, indicating the dark current damage factor

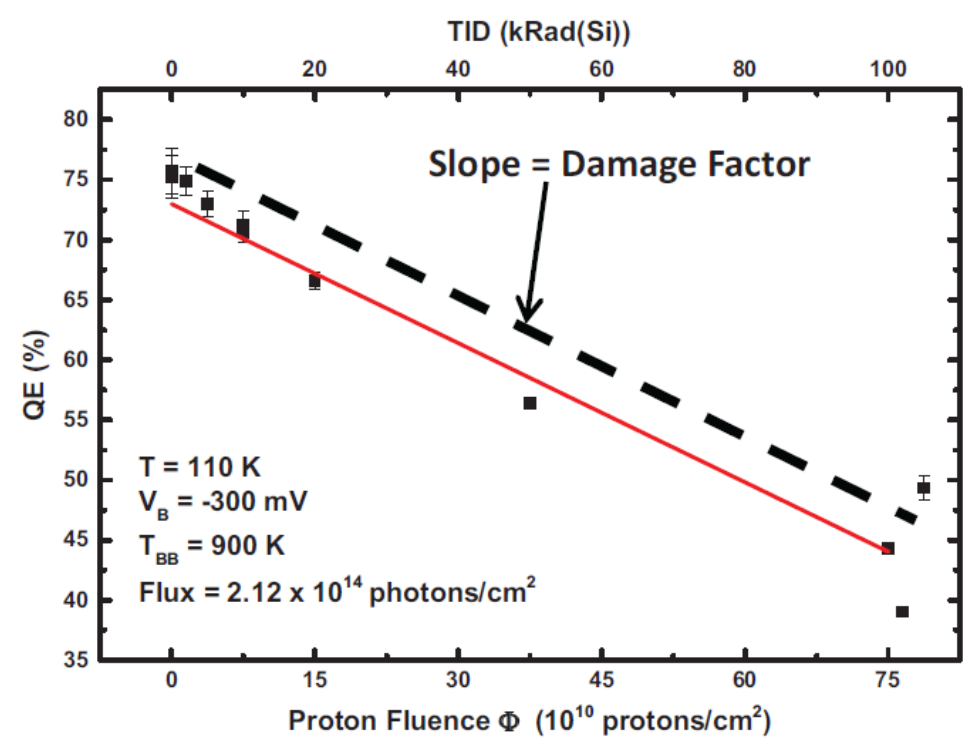

Fig. 8. Quantum efficiency as a function of proton fluence. Dashed line gives a-slope for the trend, indicating the quantum efficiency damage factor

where, $\mathrm{d} \Phi_{\mathrm{P}}(\mathrm{E}) / \mathrm{dE}$ is the expected orbit's differential proton fluence spectrum (Hubbs et al., 2007).

Comparing damage factors from different detectors may allow for the more rad-tolerant detectors to be determined (as long as several other conditions that may impact damage formation are kept constant). A connection between the damage factor and the amount of displacement damage can be established by first assuming a linear increase in the defect concentration $N_{T}$ [related to the point vacancy concentration $\mathrm{c}_{\mathrm{v}}$ introduced in Equation (40)] with $\Phi_{P}$ (which the data in (Hubbs et al., 2007; Cowan et al., 2012; Morath et al., 2015) support for the fluence ranges of interest). When dominated by SRH recombination, the minority carrier recombination lifetime $\tau_{R}$, assuming a single active recombination level, is given by:

$\frac{1}{\tau_{R}}=N_{T}\left(\Phi_{p}\right) \sigma v_{t h}$ 
where, $\sigma$ is the capture cross-section and $v_{\text {th }}$ is the carrier thermal velocity. With these assumptions in mind, the minority carrier recombination lifetime must depend on $\Phi_{P}$ according to:

$$
\frac{1}{\tau_{R}}=\frac{1}{\tau_{R o}}+K_{1 / \tau R} \Phi_{p}
$$

where, $1 / \tau_{R 0}=N_{T}(0) \sigma v_{t h}$. Based on the assumptions above and from Equations (90) and (91), a material's defect introduction rate is thus given by:

$$
\frac{d N_{T}\left(\Phi_{p}\right)}{d \Phi_{p}}=\frac{1}{\sigma v_{t h}} K_{1 / \tau R}
$$

Which means that if $\sigma v_{t h}$ is fairly constant, as expected for the fluence ranges of interest, $K_{1 / \tau R}$ is a relative measure of $d N_{T} / d \Phi_{P}$, which is a true measure of a device's defect tolerance. Thus, Equation (92) suggests that if the various performance damage factors (for diffusion-limited or SRH or trap-assisted tunneling dark current densities, lateral optical collection length, quantum efficiency, etc.), can be directly related to $K_{1 / \tau R}$, then an understanding of how they might relate to $d N_{T} / d \Phi_{P}$ and, furthermore, to each other, can be determined (Morath et al., 2015). The theory presented in this study will be used to determine the formation and dynamics of the proton-induced defect concentration and defect introduction rate that produce the damage factors that describe the degradation of sensor performance parameters. The experimentally observed increase of photo-detector dark current and decrease of quantum efficiency can be explained by defect-assisted tunneling and defect-induced capture models in Section IV, respectively.

\section{Conclusion}

In conclusion, we have proposed a multi-timescale microscopic model for fully characterizing the performance degradation of electronic and optoelectronic devices. In order to reach this goal, we will employ realistic interatomic potentials in a molecular-dynamics simulation for both the ultra-fast displacement cascade stage and the intermediate defect stabilization and cluster formation stage. This simulation will then be combined with a rate-diffusion theory for the slow defect reaction and migration stage. Additionally, with assistance from a density-functional theory for identifying defect species and their electronic properties, the calculated steady-state spatial distributions of defects and clusters will be used to study and understand the physical mechanisms that degrade electronic and optoelectronic devices, including defect-assisted resonant tunneling, reduced carrier mobility, non-radiative recombination with defects and inelastic light scattering by charged defects.
In this study, we have discussed several techniques for defect characterization. However, there are many other approaches for characterizing defect effects. These include electrical characterization techniques, such as deep-level transient spectroscopy and capacitancevoltage profiling and optical characterization techniques, such as cathodeluminescence and reflectance modulation. Physical and chemical characterization techniques can also be applied, including electron energy loss spectroscopy, (Gumbs and Horing, 1991) secondary ion mass spectrometry (Zhang et al., 2014) and chemical milling.

The molecular dynamics model presented in this study can be combined with a space-weather forecast model (Moldwin, 2008; Cooke and Katz, 1988) which predicts spatial-temporal fluxes and particle velocity distributions. With this combination of theories, the predicted irradiation conditions for particular satellite orbits allow electronic and optoelectronic devices to be specifically designed for operation in space with radiation-hardening techniques (such as self-healing and mitigation). This approach will effectively extend the lifetime of satellite onboard electronic and optoelectronic devices in nonbenign orbits and greatly reduce the cost. In addition, by improving the physical model for scintillation detectors, the accuracy of space-weather measurements will be enhanced.

\section{Acknowledgment}

Authors would like to thank the Chief Scientist of the Space Vehicles Directorate of the Air Force Research Laboratory for seed funding of this research effort.

\section{Author's Contributions}

Danhong Huang: Participated substantially in the theory of entire paper, as well as the writing/editing of the entire paper.

Fei Gao: Participated substantially in the theory and writing/editing of the sections entitled: "Introduction," "Model and Theory," and "Sink Dynamics".

D.A. Cardimona: Participated substantially in the section entitled "Application of Defect Theory" and in editing the entire paper.

C.P. Morath: Participated substantially in the theory and experiments in the section entitled "Application of Defect Theory."

V.M. Cowan: Participated substantially in the experiments in the section entitled "Application of Defect Theory."

\section{Ethics}

This article is original and contains unpublished material. The corresponding author confirms that all of 
the other authors have read and approved the manuscript and no ethical issues involved.

\section{Reference}

Abrahamson, A.A., 1969. Born-Mayer-type interatomic potential for neutral ground-state atoms with $Z=2$ to $Z=105$. Phys. Rev., 178: 76-76.

DOI: 10.1103/PhysRev.178.76

Alkauskas, A., P. Broqvist and A. Pasquarello, 2008. Charge state of the $\mathrm{O}_{2}$ molecule during silicon oxidation through hybrid functional calculations. Phys. Rev. B, 78: 161305-161305.

DOI: 10.1103/PhysRevB.78.161305

Allen, M.P. and D.J. Tildesley, 1987. Computer Simulation of Liquids. Clarendon, Oxford.

Ando, T., A.B. Fowler and F. Stern, 1982. Electronic properties of two-dimensional systems. Rev. Mod. Phys., 54: 437-437.

DOI: $10.1103 /$ RevModPhys.54.437

Brailsford, A.D., R. Bullough and M.R. Hayns, 1976. Point defect sink strengths and void-swelling. J. Nucl. Mater., 60: 246-256.

DOI: $10.1016 / 0022-3115(76) 90139-2$

Broqvist, P., A. Alkauskas and A. Pasquarello, 2008. Band alignments and defect levels in $\mathrm{SiHfO} 2$ gate stacks: Oxygen vacancy and Fermi-level pinning. Appl. Phys. Lett., 92: 132911-132911.

Bullough, B., M.R. Hayns and C.H. Woo, 1979. The sink strengths of dislocation loops and their growth in irradiated materials. J. Nucl. Mater., 84: 93-100. DOI: 10.1016/0022-3115(79)90152-1

Callaway, J., 1991. Quantum Theory of the Solid State. Academic Press, Inc., New York.

Claeys, C. and E. Simoen, 2013. Radiation Effects in Advanced Semiconductor Materials and Devices. 1st Edn., Springer Science and Business Media, Berlin, ISBN-10: 3662049740, pp: 404.

Colubov, S.I., B.N. Singh and H. Trinkaus, 2000. Defect accumulation in FCC and BCC metals and alloys under cascade damage conditions-Towards a generalisation of the production bias model. J. Nucl. Mater., 276: 78-89.

DOI: 10.1016/S0022-3115(99)00171-3

Cooke, D.L. and I. Katz, 1988. Ionization-induced instability in an electron-collecting sheath. J. Spacecraft and Rockets, 25: 132-138. DOI: $10.2514 / 3.25961$

Cowan, V.M., C.P. Morath, J.E. Hubbs, S. Myers and E. Plis et al., 2012. Radiation tolerance characterization of dual band InAs/GaSb type-II strain-layer superlattice $\mathrm{pBp}$ detectors using $63 \mathrm{MeV}$ protons. Appl. Phys. Lett., 101: 251108-251108. DOI: $10.1063 / 1.4772543$ de Walle, C.G.V. and J. Neugebauer, 2004. First-principles calculations for defects and impurities: Applications to III-nitrides. J. Appl. Phys., 95: 3851-3851. DOI: $10.1063 / 1.1682673$

Devanathan, R., T. Diaz de la Rubia and W.J. Weber, 1998. Displacement threshold energies in $\beta$-SiC. J. Nucl. Mater., 235: 47-52. DOI: 10.1016/S0022-3115(97)00304-8

Devanathan, R., W.J. Weber and F. Gao, 2001. Atomic scale simulation of defect production in irradiated 3C-SiC. J. Appl. Phys., 90: 2303-2303. DOI: $10.1063 / 1.1389523$

Doan, N.V. and G. Martin, 2003. Elimination of irradiation point defects in crystalline solids: Sink strengths. Phys. Rev. B, 67: 134107-134107. DOI: 10.1103/PhysRevB.67.134107

Drabold, D.A. and S.K. Estreicher, 2007. Theory of Defects in Semiconductors. Springer-Verlag, Berlin, Heidelberg.

Finnis, M.W. and J.E. Sinclair, 1984. A simple empirical N-body potential for transition metals. Phil. Mag. A, 50: 45-55. DOI: 10.1080/01418618408244210

Frenkel, D. and B. Smit, 2002. Understanding Molecular Simulation: From Algorithms to Applications. 1st Edn., Academic Press, San Diego, ISBN-10: 0122673514. pp: 638.

Freysoldt, C., B. Grabowski, T. Hickel and J. Neugebauer, 2014. First-principles calculations for point defects in solids. Rev. Mod. Phys., 86: 253-253. DOI: $10.1103 /$ RevModPhys.86.253

Gao, F. and W.J. Weber, 2000. Atomic-scale simulation of $50 \mathrm{keV} \mathrm{Si}$ displacement cascades in $\beta$-SiC. Phys. Rev. B, 63: 054101-054101.

DOI: 10.1103/PhysRevB.63.054101

Gao, F. and W.J. Weber, 2003a. Atomic-scale simulations of cascade overlap and damage evolution in silicon carbide. J. Mater. Res., 18: 1877-1883. DOI: 10.1557/JMR.2003.0262

Gao, F. and W.J. Weber, 2003b. Recovery of close Frenkel pairs produced by low energy recoils in SiC. J. Appl. Phys., 94: 4348-4348. DOI: 10.1063/1.1605254

Gao, F., D. Chen, W. Hu and W.J. Weber, 2010. Energy dissipation and defect generation in nanocrystalline silicon carbide. Phys. Rev. B, 81: 184101-184101. DOI: 10.1103/PhysRevB.81.184101

Gao, F., H. Xiao, X. Zu, M. POsselt and W.J. Weber, 2009. Defect-enhanced charge transfer by ion-solid interactions in $\mathrm{SiC}$ using large-scale $\mathrm{Ab}$ initio molecular dynamics simulations. Phys. Rev. Lett., 103: 027405-027405.

DOI: 10.1103/PhysRevLett.103.027405

Gao, F., H.Y. Xiao and W.J. Weber, 2011. Ab initio molecular dynamics simulations of low energy recoil events in ceramics. Nucl. Instr. Meth. Phys. Res. B, 269: 1693-1697. DOI: 10.1016/j.nimb.2011.01.131 
Garetto, D., Y.M. Randriamihaja, A. Zaka, D. Rideau and A. Schmid et al., 2012. Analysis of defect capture cross sections using non-radiative multiphonon-assisted trapping model. Solid-State Electr., 71: 74-74. DOI: 10.1016/j.sse.2011.10.024

Gear, C., 1971. Numerical Initial Value Problems in Ordinary Differential Equations. 1st Edn., PrenticeHall, Englwood Cliffs, pp: 253.

Gill, A.E., 1982. Atmosphere-ocean Dynamics. 1st Edn., Academic Press, San Diego, ISBN-10: 0122835220, pp: 662 .

Golubov, S.I., A.V. Barashev and R.E. Stoller, 2012. Radiation Damage Theory, in Comprehensive Nuclear Materials. In: Konings, R., R. Stoller, T. Allen and S. Yamanaka (Eds.), (Elsevier Ltd., Amsterdam, 2012), ch. 13.

Guinan, M.W., R.N. Stuart and R.J. Borg, 1977. Fully dynamic computer simulation of self-interstitial diffusion in tungsten. Phys. Rev. B, 15: 699-699. DOI: 10.1103/PhysRevB.15.699

Gumbs, G. and D.H. Huang, 2013. Properties of Interacting Low-Dimensional Systems. 1st Edn., John Wiley and Sons, Weinheim Wiley-VCH, ISBN-10: 3527638164. pp: 392.

Gumbs, G. and N.J.M. Horing, 1991. Plasma losses by charged particles in thin films: Effects of spatial dispersion, phonons and magnetic field. Phys. Rev. B, 43: 2119-2119. DOI: 10.1103/PhysRevB.43.2119

Gumbs, G., 1989. Inelastic electron scattering from the surfaces of semiconductor multilayers: Study of Subband structure within the quantum well. Phys. Rev. B, 39: 5186-5186. DOI: $10.1103 /$ PhysRevB.39.5186

Heald, P.T. and M.V. Speight, 1975. Point defect behavior in irradiated materials. Acta Met., 23: 1389 -1389.

Hirth, J.P. and J. Lothe, 1982. Theory of Dislocations. 2nd Edn., Krieger Publishing Company, Florida.

$\mathrm{Hu}$, S., C.H. Henager, Y. Li, F. Gao and X. Sun et al., 2012. Evolution kinetics of interstitial loops in irradiated materials: A phase-field model. Modelling Simul. Mater. Sci. Eng., 20: 015011-015011. DOI: $10.1088 / 0965-0393 / 20 / 1 / 015011$

Huang, D.H. and M.O. Manasreh, 1996. Intersubband transitions in strained $\mathrm{In}_{0.07} \mathrm{Ga}_{0.93} \mathrm{As} / \mathrm{Al}_{0.40} \mathrm{Ga}_{0.60} \mathrm{As}$ multiple quantum wells and their application to a two-colors photodetector. Phys. Rev. B, 54: 56205620. DOI: 10.1103/PhysRevB.54.5620

Huang, D.H. and P.M. Alsing, 2008. Many-body effects on optical carrier cooling in intrinsic semiconductors at low lattice temperatures. Phys. Rev. B, 78: 035206035206. DOI: 10.1103/PhysRevB.78.035206

Huang, D.H. and S.K. Lyo, 1999. Photoluminescence spectra of n-doped double quantum wells in a parallel magnetic field. Phys. Rev. B, 59: 7600-7600.

DOI: 10.1103/PhysRevB.59.7600
Huang, D.H. and S.X. Zhou, 1988. Theoretical investigation of collective excitations in $\mathrm{HgTe} / \mathrm{CdTe}$ superlattices. II. Intersubband excitation and effects of magnetic field and electron-phonon coupling. Phys. Rev. B, 38: 13069-13069.

DOI: 10.1103/PhysRevB.38.13069

Huang, D.H., A. Singh and D.A. Cardimona, 1999. Effects of structural disorders on sequential tunneling in multiple quantum wells. Phys. Lett. A, 259: 488-488. DOI: 10.1016/S0375-9601(99)00473-9

Huang, D.H., A. Singh and D.A. Cardimona, 2000. Change of microscopic tunneling on a macroscopic time scale: Current surge model. J. Appl. Phys., 87: 2427-2427. DOI: $10.1063 / 1.372196$

Huang, D.H., C. Rhodes, P.M. Alsing and D.A. Cardimona, 2006. Effects of longitudinal field on transmitted near field in doped semi-infinite semiconductors with a surface conducting sheet. J. Appl. Phys., 100: 113711-113711.

DOI: $10.1063 / 1.2388651$

Huang, D.H., G. Gumbs and M. Pepper, 2008. Effects of inelastic capture, tunneling escape and quantum confinement on surface acoustic wave-dragged photocurrents in quantum wells. J. Appl. Phys., 103: 083714-083714. DOI: 10.1063/1.2890403

Huang, D.H., P.M. Alsing, T. Apostolova and D.A. Cardimona, 2005a. Coupled energy-drift and forcebalance equations for high-field hot-carrier transport. Phys. Rev. B, 71: 195205-195205. DOI: 10.1103/PhysRevB.71.195205

Huang, D.H., P.M. Alsing, T. Apostolova and D.A. Cardimona, 2005b. Effect of photon-assisted absorption on the thermody-namics of hot electrons interacting with an intense optical field in bulk GaAs. Phys. Rev. B, 71: 045204-045204.

DOI: 10.1103/PhysRevB.71.045204

Hubbs, J.E., P.W. Marshall, C.J. Marshall, M.E. Gramer and D. Maestas et al., 2007. Lateral diffusion length changes in $\mathrm{HgCdTe}$ detectors in a proton environment. IEEE Trans. Nucl. Sci., 54: 2435-2443. DOI: 10.1109/TNS.2007.910329

Jiang, C., N. Swaminathan, J. Deng, D. Morgan and I. Szlufarsk, 2014. Effect of grain boundary stresses on sink strength. Mater. Res. Lett., 2: 100-106. DOI: $10.1080 / 21663831.2013 .871588$

Jim'enez-Molinos, F., A. Palma, F. G'amiz, J. Banqueri and J.A. Lopez-Villanueva, 2001. Physical model for trap-assisted inelastic tunneling in metal-oxidesemiconductor structures. J. Appl. Phys., 90: 33963396. DOI: $10.1063 / 1.1398603$

Kinchin, G.H. and R.S. Pease, 1955. The displacement of atoms in solids by radiation. Rep. Prog. Phys., 18: 1-1. DOI: $10.1088 / 0034-4885 / 18 / 1 / 301$ 
Kohanoff, J., 2006. Electronic Structure Calculations for Solids and Molecules. Cambridge Press.

Kohn, W. and L.J. Sham, 1965. Self-consistent equations including exchange and correlation effects. Phys. Rev., 140: A1133-A1133. DOI: 10.1103/PhysRev.140.A1133

Li, Y., S. Hu, X. Sun, F. Gao and C.H. Henager et al., 2010. Phase-field modeling of void migration and growth kinetics in materials under irradiation and temperature field. J. Nucl. Mater., 407: 119-125. DOI: 10.1016/j.jnucmat.2010.09.048

Maksimov, L.A. and A.I. Ryazanov, 1980. Kinetic equation for vacancion pores. Pore lattice as a dissipative structure stable under irradiation conditions. Sov. Phys. JETP, 52: 1170-1170.

Mehrer, H., 2007. Diffusion in Solids: Fundamentals, Methods, Materials, Diffusion-Controlled Processes. 1st Edn., Springer Science and Business Media, Berlin, ISBN-10: 354071488X. pp: 672.

Moldwin, M., 2008. An Introduction to Space Weather. 1st Edn., Cambridge University Press, New York, ISBN-10: 1139467859.

Morath, C.P., V.M. Cowan, L.A. Treider, G. Jenkins and J.E. Hubbs, 2015. Proton irradiation effects on the performance of III-V-based, unipolar barrier infrared detectors. IEEE Trans. Nucl. Sci.

Murty, K.L. and I. Charit, 2013. An Introduction to Nuclear Materials: Fundamentals and Applications. 1 st Edn., John Wiley and Sons, Weinheim, ISBN-10: 3527412018, pp: 382.

Nord, J., K. Nordlund, J. Keinonen and K. Albe, 2003. Molecular dynamics study of defect formation in GaN cascades. Nucl. Instr. Meth. Phys. Res. B, 202: 93-99. DOI: 10.1016/S0168-583X(02)01839-6

Nordlund, K., J. Peltola, J. Nord, J. Keinonen and R.S. Averback, 2001. Defect clustering during ion irradiation of GaAs: Insight from molecular dynamics simulations. J. Appl. Phys., 90: 1710-1710. DOI: $10.1063 / 1.1384856$

Norskov, J.K. and N. Lang, 1980. Effective-medium theory of chemical binding: Application to chemisorption. Phys. Rev. B, 21: 2131-2131. DOI: 10.1103/PhysRevB.21.2131

Olander, D.R., 1976. Fundamental Aspects of Nuclear Reactor Fuel Elements. 1st Edn., Technical Information Center, Oak Ridge, ISBN-10: 0870790315, pp: 551.

Platzman, P.M. and P.A. Wolff, 1973. Waves and Interactions in Solid State Plasmas. 1st Edn., Academic Press, New York, ISBN-10: 012607772X, pp: 293.

Posselt, M., F. Gao and D. Zwicker, 2005. Atomistic study of the migration of di- and tri-interstitials in silicon. Phys. Rev. B, 71: 245202-245202.

DOI: 10.1103/PhysRevB.71.245202
Posselt, M., F. Gao and H. Bracht, 2008. Correlation between self-diffusion in $\mathrm{Si}$ and the migration mechanisms of vacancies and self-interstitials: An atomistic study. Phys. Rev. B, 78: 035208035208. DOI: 10.1103/PhysRevB.78.035208

Ridley, B.K., 1978. Multiphonon, non-radiative transition rate for electrons in semiconductors and insulators. J. Phys. C: Solid State Phys., 11: 2323-2323. DOI: $10.1088 / 0022-3719 / 11 / 11 / 023$

Rong, Z., F. Gao and W.J. Weber, 2007. Monte Carlo simulations of defect recovery within a $10 \mathrm{keV}$ collision cascade in 3CSiC. J. Appl. Phys., 102: 103508-103508. DOI: 10.1063/1.2812701

Rubia, T.D.D.L. and G.H. Gilmer, 1995. Structural transformations and defect production in ion implanted silicon: A molecular dynamics simulation study. Phys Rev. Lett., 74: 2507-2507.

DOI: 10.1103/PhysRevLett.74.2507

Rutherford, E., 1911. The scattering of a and $\beta$ particles by matter and the structure of the atom. Phil. Mag., 21: 669-669.

Semenov, A.A. and C.H. Woo, 2002. Void nucleation at elevated temperatures under cascade-damage irradiation. Phys. Rev. B, 66: 024118-024118. DOI: 10.1103/PhysRevB.66.024118

Sigmund, P., 2006. Particle Penetration and Radiation Effects: General Aspects and Stopping of Swift Point Charges. 1st Edn., Springer Science and Business Media, Berlin Heidelberg, ISBN-10: 3540317139. pp: 437.

Singh, B.N. and S.J. Zinkle, 1993. Defect accumulation in pure fcc metals in the transient regime: A review. J. Nucl. Mater., 206: 212-229. DOI: 10.1016/0022-3115(93)90125-I

Stievenard, D., X. Letartre and M. lannoo, 1992. Defectassisted resonant tunneling: A theoretical model. Appl. Phys. Lett., 61: 1582-1582.

DOI: $10.1063 / 1.107503$

Strour, J.R., C.J. Marshall and P.W. Marshall, 2003. Review of displacement damage effects in silicon devices. IEEE Trans. Nucl. Sci., 50: 653-670. DOI: $10.1109 /$ TNS.2003.813197

Terentyev, D., C. Lagerstedt, P. Olsson, K. Nordlund and J. Wallenius et al., 2006. Effect of the interatomic potential on the features of displacement cascades in $\alpha-\mathrm{Fe}$ : A molecular dynamics study. J. Nucl. Mater., 351: 65-67.

DOI: 10.1016/j.jnucmat.2006.02.020

Tersoff, J., 1988. New empirical approach for the structure and energy of covalent systems. Phys. Rev. B, 37: 6991-6991. DOI: 10.1103/PhysRevB.37.6991

Tschopp, M.A., K.N. Solanki, F. Gao, X. Sun and M.A. Khaleel et al., 2012. Probing grain boundary sink strength at the nanoscale: Energetics and length scales of vacancy and interstitial absorption by grain boundaries in $\alpha$-Fe. Phys. Rev. B, 85: 064108-064108. DOI: 10.1103/PhysRevB.85.064108 
Tsen, K.T., R.K. Willardson and E.R. Weber, 2001. Ultrafast Physical Processes in Semiconductors. 1st Edn., Elsevier, San Diego, ISBN-10: 0080540953, pp: 468.

Tuckerman, M.E. and G.J. Martyna, 2000. Understanding modern molecular dynamics: Techniques and applications. J. Phys. Chem. B, 104: 159-159. DOI: $10.1021 /$ jp992433y

Tuomisto, F. and I. Makkonen, 2013. Defect identification in semiconductors with positron annihilation: Experiment and theory. Rev. Mod. Phys., 85: 1583-1583.

DOI: 10.1103/RevModPhys.85.1583

Waite, T.R., 1957. Theoretical treatment of the kinetics of diffusion-limited reactions. Phys. Rev., 107: 463-463. DOI: 10.1103/PhysRev.107.463

Was, G.S., 2007. Fundamentals of Radiation Materials Science: Metals and Alloys. 1st Edn., Springer Science and Business Media, Berlin, ISBN-10: 3540494715. pp: 827.

Weatherford, T.R. and W.T. Anderson, 2003. Historical perspective on radiation effects in III-V devices. IEEE Trans. Nucl. Sci., 50: 704-710.

DOI: $10.1109 /$ TNS.2003.813124

Weertman, J., 1965. The Peach-Koehler equation for the force on a dislocation modified for hydrostatic pressure. Phil. Mag., 11: 1217-1223.

DOI: $10.1080 / 14786436508224930$

Wiedersich, H., P.R. Okamoto and N.Q. Lam, 1979. A theory of radiation-induced segregation in concentrated alloys. J. Nucl. Mater., 83: 98-108. DOI: 10.1016/0022-3115(79)90596-8

Zhang, C., G. Gumbs and D.H. Huang, 1991. Theory of the Raman-scattering intensity and line shape of a quantum-dot superlattice. Phys. Rev. B, 44: 10744-10744. DOI: 10.1103/PhysRevB.44.10744

Zhang, Y., M.L. Crespillo, H. Xue, K. Jin and C.H. Chen et al., 2014. New ion beam materials laboratory for materials modification and irradiation effects research. Nucl. Instr. Meth. B, 338: 19-30.

DOI: $10.1016 /$ j.nimb.2014.07.028 